\title{
A low-luminosity core collapse supernova very similar to SN 2005cs
}

\author{
Zoltán Jäger Jr. ${ }^{1,2}$, József Vinkó $2,3,5$, Barna I. Bíró ${ }^{1}$, Tibor Hegedüs ${ }^{1}$, \\ Tamás Borkovits ${ }^{1,3}$, Zoltán Jäger Sr. ${ }^{1}$, Andrea P. Nagy²,3, László Molnár ${ }^{3,4,5}$, \\ Levente Kriskovics 3,5
}

\footnotetext{
${ }^{1}$ Baja Astronomical Observatory of the University of Szeged, Szegedi út KT766, H-6500 Baja, Hungary

${ }^{2}$ Department of Optics and Quantum Electronics, University of Szeged, Dóm tér 9, H-6720 Szeged, Hungary

${ }^{3}$ Konkoly Observatory, Research Center for Astronomy and Earth Sciences, Konkoly Thege Miklós út 15-17., Budapest H-1121, Hungary

${ }^{4}$ CSFK Lendület Near-Field Cosmology Research Group, Konkoly Thege 15-17, H-1121 Budapest, Hungary

${ }^{5}$ ELTE Eötvös Loránd University, Institute of Physics, Pázmány Péter sétány 1/A, Budapest, 1117 Hungary
}

Accepted 2020 June 9. Received 2020 June 9; in original form 2019 September 24

\begin{abstract}
We present observations and analysis of PSN J17292918+7542390, a lowluminosity Type II-P supernova (LL SN IIP). The observed sample of such events is still low, and their nature is still under debate. Such supernovae are similar to SN 2005cs, a well-observed low-luminosity Type II-P event, having low expansion velocities, and small ejected ${ }^{56} \mathrm{Ni}$ mass. We have developed a robust and relatively fast Monte-Carlo code that fits semi-analytic models to light curves of core collapse supernovae. This allows the estimation of the most important physical parameters, like the radius of the progenitor star, the mass of the ejected envelope, the mass of the radioactive nickel synthesized during the explosion, among others. PSN J17292918+7542390 has $R_{0}=91_{-70}^{+119} \cdot 10^{11} \mathrm{~cm}, M_{\mathrm{ej}}=9.89_{-1.00}^{+2.10} M_{\odot}, E_{\mathrm{kin}}=0.65_{-0.18}^{+0.19}$ foe, $v_{\mathrm{exp}}=3332_{-347}^{+216}$ $\mathrm{km} \mathrm{s}^{-1}$, for its progenitor radius, ejecta mass, kinetic energy and expansion velocity, respectively. The initial nickel mass of the PSN J17292918+7542390 turned out to be $1.55_{-0.70}^{+0.75} \cdot 10^{-3} M_{\odot}$. The measured photospheric velocity at the earliest observed phase is $7000 \mathrm{~km} \mathrm{~s}^{-1}$. As far as we can tell based on the small population of observed low-luminosity Type II-P supernovae, the determined values are typical for these events.
\end{abstract}

Key words: Supernovae: individual: PSN J17292918+7542390, 2005cs, 1997D, 1999em, 2004et, 2009md, 2012aw - instrumentation: photometers techniques: photometric - methods: data analysis - methods: numerical

\section{INTRODUCTION}

Core-collapse supernovae (CC SNe) are thought to originate from stars having $M_{Z A M S}>8 M_{\odot}$, following the collapse of their iron core to a neutron star or black hole (Burrows 2013; Burrows et al. 2019). Stars that retain their massive hydrogen-rich envelope produce Type II-P and II-L SNe, showing strong $\mathrm{H}$ features in their spectra and a plateau (with a wide range in duration) in their optical light curves.In the past 20 years the existence of a subclass of low-luminosity (LL) Type II-P SNe has been recognized (Pastorello et al. 2004; Spiro et al. 2014). These events have absolute magnitudes fainter by $\sim 2-3$ mag than regular Type II-P SNe (absolute magnitude between -16 - -19 mag), have lower expansion velocities, and seem to produce less amount of radioactive nickel during explosion.

The prototype of this group was SN 1997D (Turatto et al. 1998), which unfortunately was discovered at later phase giving high uncertainties in the explosion epoch (Benetti et al. 2001). The origin of these events is still under debate. 
They may arise from a moderate mass $\left(\sim 9 M_{\odot}\right)$ star, or from a massive star $\left(\sim 25 M_{\odot}\right)$ that failed to explode entirely (Turatto et al. 1998; Chugai \& Utrobin 2000; Kitaura, Janka, Hillebrandt 2006). Light-curve modeling and progenitor identification from pre-explosion images (e.g. for $2005 \mathrm{cs}$ : $M_{0}=6-13 M_{\odot}$ by Maund et al. 2005; Li et al. 2006; Eldridge et al. 2007) suggest they are likely to arise from moderate mass red supergiant (RSG) stars (Pumo et al. 2017; Lisakov et al. 2018 and references therein) with masses between 8-12 $M_{\odot}$. However the massive star scenario may also be possible: SN 2016bkv had an ejected mass between 16-19 $M_{\odot}$ (Nakaoka et al. 2018). They might also be electron capture $\mathrm{SNe}$, where, instead of a collapsing Fe core, an O$\mathrm{Ne}-\mathrm{Mg}$ core captures the electrons, which leads to a subsequent collapse (see Kitaura, Janka, Hillebrandt 2006; Wanajo et al. 2008). However iron core collapse is also a possibility: for example, SN 1997D had more likely an iron core rather than $\mathrm{O}-\mathrm{Ne}-\mathrm{Mg}$ core (Jerkstrand et al. 2017). The sample of sub-luminous SNe is still small, they represent only $5 \%$ of all the Type II SNe events (Pastorello et al. 2004), so any new observed event may be important to investigate.

Assuming a Salpeter initial mass function (IMF) with an exponent of $2.3,42 \%$ of the massive stars are born with masses between $8-12 M_{\odot}$, and $15 \%$ in the range $8-9 M_{\odot}$. The range may be even narrower for these low-luminous events, but we probably miss many of them due to their fainter absolute brightness.

PSN J17292918+7542390, hereafter SN-NGC6412, a LL Type II-P SN, was discovered on 2015-07-10 (57213 MJD) by Ron Arbour amateur astronomer (Tomasella et al. 2015). A single spectrum was made one day after the discovery epoch (57214 MJD) by Tomasella et al. ( 2015). No additional data have been published for this event

In this paper we present photometric observations of SN-NGC6412 taken at two observatories in Hungary. This object turned out to be a low luminosity IIP SN with low velocities, and small ejected ${ }^{56} \mathrm{Ni}$ mass. Even though our follow-up observations missed the end of the plateau, the rarity of such events may still make the data and the analysis valuable.

This paper also contains the modeling of the bolometric light curve (LC) of SN-NGC6412 using a semianalytic model. Such simple light curve (LC) models have been developed as early as the 1980's by Arnett ( 1980, 1982) to explain and fit bolometric light curves of supernovae. Later they were further improved by the inclusion of numerical computations of certain aspects, thus becoming semi-analytic (Arnett \& Fu 1989; Popov 1993). These models assume spherical symmetry and do not adequately model the initial transient behavior at early stages $(\mathrm{t}<20$ days), which obviously makes them inferior compared to detailed hydrodynamical models; nevertheless, they are useful to derive estimates or constraints of basic parameters like the explosion energy, ejected mass and initial radius, without the high computational resources demanded by the latter.
Applications of the code so far indicate that it can model IIP LC particularly well, while other SN types may have further physical mechanisms which is not considered in this model: Circumstellar medium (CSM) interaction for IIn (narrow emission line in spectra) and IIL (no significant plateau present), and binary companion for IIb, Ib and Ic (weak or no $\mathrm{H}$ and silicon present in spectra) (see Nagy 2018). They can also give first-order approximations in cases of limited observational information, for example when only photometric time series are available for a particular SN. Fitting the parameters of such models to the light curve has been traditionally made "by hand" using a trial and error approach. Strong correlations between the physical parameters, however, make this procedure complicated (Arnett \& Fu 1989; Nagy et al. 2014). This approach also lacks a firm estimate of the parameter uncertainties. However, in the age of high computing powers, a method of Markov Chain Monte Carlo (MCMC) sampling becomes feasible (Metropolis et al. 1953; Hastings 1970). MCMC is a well-established technique for constraining parameters from observed data, and especially suited for cases when the parameter space has a high dimensionality (Gilks et al. 1996). The sampling maps the whole parameter space based on the joint posterior probability distribution of all the parameters. It has the nice ergodic property which allows the various integrals over the parameter space (mean value, standard deviation, and percentiles in particular) to be computed as simple sums over the chain elements. Therefore it makes possible to provide not only best estimates of the parameters, but also confidence intervals for them (leading to uncertainty determinations); furthermore, correlations between the various parameters can also be revealed.

In Section 2 we describe the details of observations of SN-NGC6412, and then we make an approximation for the dust extinction in Section 3. In Section 4 we compare the light curve of the SN-NGC6412 with other SNe. Section 5 presents the analysis of SN-NGC6412 spectrum. In Section 6 we fit diluted blackbody radiation to the photometric filters to determine the temperatures and photometric radii at various times of observation. Sections $7-9$ present the bolometric light curve calculation, and the MCMC analysis of SN-NGC6412. In Section 10 we present the results, and finally Section 11 summarizes the main conclusions of this paper. The Appendix contains a technical description of the MCMC fitting program used in the analysis.

\section{PHOTOMETRY}

For the photometric monitoring of SN-NGC6412 we used two optical telescopes. One of them is a $50 \mathrm{~cm}$ telescope of Baja Observatory, equipped with SDSS griz filters. The other is the $60 / 90 \mathrm{~cm}$ Schmidt telescope located at Piszkéstető Mountain Station of Konkoly Observatory, and uses Johnson BVRI filters. Our photometric monitoring of SN-NGC6412 began on 2015-0712, 2 days after its discovery, and continued for $\sim 100$ days up to the end of the plateau phase. After emerging 
from solar conjunction, the last observation was made after 250 days with both telescopes, however, by that time the SN became very dim.

The data were reduced with standard prescription in IRAF environment. We used Point Spread Function (PSF) photometry to obtain the magnitude values. Nearby fields covered by the SDSS survey were observed during the same nights as the SN and the SDSS DR15 catalog ${ }^{1}$ (Albareti et al. 2017) was used to determine standard magnitudes of stars in the target field. (The catalog unfortunately does not cover the field of NGC 6412 containing the supernova.) These magnitudes were then used to tie the magnitudes of the SN to standard systems. The uncertainties were computed using standard propagation of errors, the major contribution coming from the standard calibration. Because of the two different filter systems, one of them must be converted to the other using empirical relations measured by Jordi et al. ( 2006). We chose to convert the SDSS griz to Johnson BVRI. The calibrated SN magnitudes are reported in Table 1 . The SN appeared in a brighter region (possibly a H II region) which has nonnegligible contribution to the measured brightness of the SN. This region was observed $\sim 2$ years after the peak $(\mathrm{MJD}=57834.5)$ when the SN already dimmed below the detection limit, and its fluxes were subtracted from the SN fluxes.

The last epoch when the $\mathrm{SN}$ was detected (MJD = 57464.5 ) was $\sim 254$ days after explosion (see Section 6 for a discussion of the date of explosion $\left.\left(t_{0}\right)\right)$. At this phase the SN was only slightly $(0.2-0.3 \mathrm{mag})$ brighter than the underlying $\mathrm{H}$ II region in all bands, which makes the uncertainty of the measured brightnesses quite high. Nevertheless, the faintness of SN-NGC6412 at such a late phase suggests low amounts of initial nickel mass.

Fig. 1 shows the LC of SN-NGC6412 in BVRIbands, converted to absolute magnitudes (see Section 4), and compared to those of other Type II-P SNe. The presence of the plateau as well as the low absolute magnitudes make SN-NGC6412 a member of the LL Type II-P SN subclass.

\section{ESTIMATION OF DUST EXTINCTION}

The interstellar reddening due to the Milky Way dust in the direction of SN-NGC6412 is $E(B-V)=0.035 \mathrm{mag}$ acquired from the NASA Extragalactic Database (NED; Schlafly \& Finkbeiner 2011).

The total extinction that includes the effect of intergalactic dust as well as the dust within the host galaxy can be estimated from the equivalent width (EW) of the $\mathrm{Na} \mathrm{D}$ line, which correlates with $E(B-V)$ (see Poznanski et al. 2012 for more information). Combining the unresolved $\mathrm{Na} \mathrm{D}_{1}$ and $\mathrm{D}_{2}$ features one can get

$$
\log _{10} E(B-V)=1.17 \cdot \mathrm{EW}\left(\mathrm{D}_{1}+\mathrm{D}_{2}\right)-1.85 \pm 0.08
$$

Examining the observed spectrum of SN-NGC6412

\footnotetext{
1 http://skyserver.sdss.org/dr9/en/tools/chart/navi.asp
}

(see Section 5) around the Na D line (Fig. 2 top right panel), shows no features exceeding the noise level significantly. The panel shows the Na D line of the host and the milky way in the spectra. They are not in the same position due the redshift. The weak features appearing in the spectrum are probably noise, implying low host extinction, which is in agreement with the fact that the galaxy is seen face-on. Therefore we neglect the intergalactic and host galaxy extinction, and adopt $E(B-V)=0.035 \mathrm{mag}$.

\section{COMPARISON WITH SN 2005CS AND OTHER SNE}

A comparison of the LCs of SN-NGC6412 and SN 2005cs (Dessart et al. 2008; Pastorello et al. 2006; Pastorello et al. 2009) reveals some remarkable similarities, as shown in Fig. 1, and also in Table 2 of the adopted values.

Despite its relative proximity, the distance to the host galaxy, NGC 6412, is quite uncertain. The NASA Extragalactic Database $^{2}$ (NED) lists several values determined from various methods. Bottinelli et al. ( 1984, 1986) acquired distances between 12.4 and $14.9 \mathrm{Mpc}$ with the Tully-Fisher (T-F) method, but with quite high $(\sim 4-5 \mathrm{Mpc})$ uncertainty. They assumed $H_{0}=103$ $\mathrm{km} \mathrm{s}^{-1} \mathrm{Mpc}^{-1}$ for the value of the Hubble-constant. On the other hand, Tully \& Fisher (1988) obtained $23.5 \pm 4.8 \mathrm{Mpc}$ from the same method, assuming $H_{0}=75$ $\mathrm{km} \mathrm{s}^{-1} \mathrm{Mpc}^{-1}$. To our knowledge, no other redshift independent distance estimate is available for NGC 6412.

The issue with this inhomogeneous dataset is twofold. First, they are tied to different calibrations relating the relative distances and the absolute scale, reflected by the different values of the Hubble-constant. Second, NGC 6412 is a face-on galaxy, which renders the measured rotation velocities uncertain.

Using a more recently determined Hubble-constant may reduce the systematic offset between the various calibrations. At present there is a well-known tension between the $H_{0}$ parameters measured either in the local Universe or at high redshifts: from CMB fluctuations $H_{0}=67.37 \pm 0.54 \mathrm{~km} \mathrm{~s}^{-1} \mathrm{Mpc}^{-1}$ was obtained by the Planck mission (Planck Collaboration 2018), while Cepheids and Type Ia supernovae in the local Universe resulted in $H_{0}=73.48 \pm 1.66 \mathrm{~km} \mathrm{~s}^{-1} \mathrm{Mpc}^{-1}$ (Riess et al. 2020). If we adopt the mean of these two measurements, $H_{0} \sim 71 \mathrm{~km} \mathrm{~s}^{-1} \mathrm{Mpc}^{-1}$, then the T-F distances by Bottinelli et al. ( 1984,1986$)$ will increase to values between 18 and $21.6 \mathrm{Mpc}$, while the distance given by Tully \& Fisher ( 1988 ) will be $\sim 24.8 \mathrm{Mpc}$, thus, reducing the gap between the various distance measurement results.

NED also gives redshift-dependent distances based on the kinematics of NGC 6412. The recession velocity corrected for the infall to the Virgo cluster (Mould et al. $2000)$ is $v_{\text {Virgo }}=1715 \pm 18 \mathrm{~km} \mathrm{~s}^{-1}$, which gives $D_{\text {kin }} \sim 24.1 \pm 0.25 \mathrm{Mpc}$ adopting $H_{0}=71 \mathrm{~km} \mathrm{~s}^{-1} \mathrm{Mpc}^{-1}$

2 https://ned.ipac.caltech.edu 
Table 1. BVRI photometry of SN-NGC6412. All data are transformed to the Johnson-Cousins BVRI system. No further corrections have been applied. The data taken on the last epoch $($ at $+625 \mathrm{~d})$ were used to correct for the contamination from the underlying $\mathrm{H}$ II region.

\begin{tabular}{|c|c|c|c|c|c|c|c|c|c|c|}
\hline MJD & Phase & B & B err & V & V err & $\mathbf{R}$ & $R$ err & I & I err & Inst. \\
\hline 57215.5 & 5.5 & 16.948 & 0.027 & 16.863 & 0.029 & 16.792 & 0.012 & 16.833 & 0.012 & 1 \\
\hline 57216.3 & 6.3 & 16.916 & 0.025 & 16.832 & 0.024 & 16.755 & 0.012 & 16.665 & 0.032 & 1 \\
\hline 57219.3 & 9.3 & 16.876 & 0.031 & 16.708 & 0.022 & 16.578 & 0.012 & 16.517 & 0.026 & 1 \\
\hline 57220.5 & 10.5 & 16.895 & 0.018 & 16.734 & 0.015 & 16.611 & 0.006 & 16.565 & 0.009 & 1 \\
\hline 57221.0 & 11.0 & 16.862 & 0.049 & 16.649 & 0.020 & 16.574 & 0.018 & 16.412 & 0.038 & 2 \\
\hline 57222.5 & 12.5 & 16.966 & 0.036 & 16.705 & 0.017 & 16.520 & 0.008 & 16.497 & 0.017 & 1 \\
\hline 57223.0 & 13.0 & 16.911 & 0.039 & 16.667 & 0.017 & 16.566 & 0.018 & 16.336 & 0.029 & 2 \\
\hline 57224.0 & 14.0 & 16.872 & 0.033 & 16.583 & 0.013 & 16.471 & 0.011 & 16.309 & 0.024 & 2 \\
\hline 57226.0 & 16.0 & 16.887 & 0.051 & 16.624 & 0.020 & 16.509 & 0.016 & 16.250 & 0.037 & 2 \\
\hline 57231.8 & 21.8 & 17.080 & 0.025 & 16.564 & 0.011 & 16.338 & 0.012 & 16.163 & 0.028 & 2 \\
\hline 57235.0 & 25.0 & 17.268 & 0.080 & 16.737 & 0.027 & 16.487 & 0.011 & 16.210 & 0.032 & 2 \\
\hline 57236.0 & 26.0 & 17.200 & 0.050 & 16.722 & 0.019 & 16.495 & 0.016 & 16.201 & 0.041 & 2 \\
\hline 57239.0 & 29.0 & 17.299 & 0.019 & 16.740 & 0.010 & 16.486 & 0.012 & 16.272 & 0.023 & 2 \\
\hline 57242.0 & 32.0 & 17.434 & 0.024 & 16.778 & 0.010 & 16.466 & 0.008 & 16.196 & 0.016 & 2 \\
\hline 57243.0 & 33.0 & 17.343 & 0.027 & 16.767 & 0.011 & 16.487 & 0.009 & 16.162 & 0.016 & 2 \\
\hline 57244.0 & 34.0 & 17.345 & 0.026 & 16.737 & 0.010 & 16.452 & 0.007 & 16.192 & 0.013 & 2 \\
\hline 57244.8 & 34.8 & 17.425 & 0.054 & 16.748 & 0.020 & 16.427 & 0.014 & 16.158 & 0.023 & 2 \\
\hline 57245.8 & 35.8 & 17.366 & 0.024 & 16.735 & 0.015 & 16.436 & 0.020 & 16.162 & 0.039 & 2 \\
\hline 57246.8 & 36.8 & 17.418 & 0.038 & 16.765 & 0.015 & 16.455 & 0.012 & 16.187 & 0.026 & 2 \\
\hline 57248.8 & 38.8 & 17.364 & 0.031 & 16.727 & 0.012 & 16.425 & 0.011 & 16.160 & 0.037 & 2 \\
\hline 57252.8 & 42.8 & 17.451 & 0.016 & 16.726 & 0.008 & 16.372 & 0.009 & 16.047 & 0.019 & 2 \\
\hline 57259.8 & 49.8 & 17.513 & 0.055 & 16.722 & 0.018 & 16.330 & 0.009 & 15.978 & 0.048 & 2 \\
\hline 57263.8 & 53.8 & 17.510 & 0.032 & 16.729 & 0.016 & 16.334 & 0.018 & 15.933 & 0.039 & 2 \\
\hline 57265.8 & 55.8 & 17.520 & 0.038 & 16.716 & 0.017 & 16.316 & 0.017 & 15.951 & 0.033 & 2 \\
\hline 57267.0 & 57.0 & 17.513 & 0.043 & 16.704 & 0.016 & 16.310 & 0.010 & 15.994 & 0.023 & 2 \\
\hline 57267.3 & 57.3 & No data & No data & 16.723 & 0.011 & 16.307 & 0.014 & 15.946 & 0.020 & 1 \\
\hline 57268.3 & 58.3 & 17.570 & 0.014 & 16.721 & 0.007 & 16.295 & 0.011 & 15.915 & 0.025 & 1 \\
\hline 57275.3 & 65.3 & 17.670 & 0.012 & 16.716 & 0.009 & 16.286 & 0.006 & 15.900 & 0.022 & 1 \\
\hline 57318.3 & 108.3 & 18.073 & 0.272 & 16.852 & 0.008 & 16.334 & 0.014 & 15.966 & 0.053 & 1 \\
\hline 57464.5 & 254.5 & 19.393 & 0.236 & 18.877 & 0.134 & No data & No data & No data & No data & 2 \\
\hline 57464.5 & 254.5 & 19.335 & 0.133 & 19.141 & 0.127 & 18.606 & 0.090 & 18.222 & 0.097 & 1 \\
\hline 57834.5 & 624.5 & 19.375 & 0.092 & 19.404 & 0.104 & 19.087 & 0.080 & 18.656 & 0.098 & 1 \\
\hline
\end{tabular}

${ }^{1}$ 60/90 cm Schmidt telescope, BVRI filters, Konkoly Observatory, Piszkestető, Hungary

$250 \mathrm{~cm}$ RC-telescope, SDSS griz filters, Baja Observatory, Baja, Hungary

as above. On the other hand, the velocity with respect to the 3K Cosmic Background Radiation (CMB), $v_{C M B}=1262 \pm 4 \mathrm{~km} \mathrm{~s}^{-1}$ (Fixsen et al. 1996) would result in $D_{C M B} \sim 17.8 \pm 1.3 \mathrm{Mpc}$. These estimates have about the same amount of discrepancy $(\sim 6 \mathrm{Mpc})$ as the results from the T-F method listed above.

Unfortunately, the various distance measurement methods that use the SN itself cannot be applied in this case. The Expanding Photosphere Method (EPM, Kirshner \& Kwan 1974) needs velocity information, which is not available here, because of the single spectrum of SN-NGC6412 observed at very early phase. The application of that spectrum would give a very high ( $20 \mathrm{Mpc}$ ) uncertainty for the EPM-distance. The Standard Candle Method for Type II-P SNe (Hamuy \& Pinto 2002) is also less reliable for LL SNe II.

After finding no additional constraint, we decided to adopt the $D_{C M B} \sim 17.8 \mathrm{Mpc}$ distance, that is also close to the rescaled T-F result from Bottinelli et al. (1984). The motivation for choosing this value is that in this case the absolute light curves of SN-NGC6412 are overlap with those of SN 2005cs. While this is not necessarily true, as LL SNe II display a range of absolute magnitudes, this is the only additional constraint that can be applied in the distance estimate. If the longer, $D \sim 24 \mathrm{Mpc}$ distance were the true one, then SN-NGC6412 would be 0.6 mag brighter, which must be kept in mind while comparing the absolute magnitudes to those of other SNe II-P.

Because of the remarkable similarities, we used the very well-observed SN 2005cs as the main reference for comparative analysis. SN $2005 \mathrm{cs}$ was also a sub-luminous, ${ }^{56} \mathrm{Ni}$-poor, low-energy Type II-P SN (Pastorello et al. 2009) similar to SN-NGC6412. While the B band fluxes evolved slightly differently, the V, R and I band light curves look very similar. Although the brightnesses at the earliest phases are a bit fainter for SN-NGC6412, the luminosities of the plateau are the same. The length of SN-NGC6412 plateau is uncertain due to the lack of observations.

The light curves are also compared with those of other Type II-P SNe collected from the literature. One of them is SN 1997D (Turatto et al. 1998, Benetti et al. 

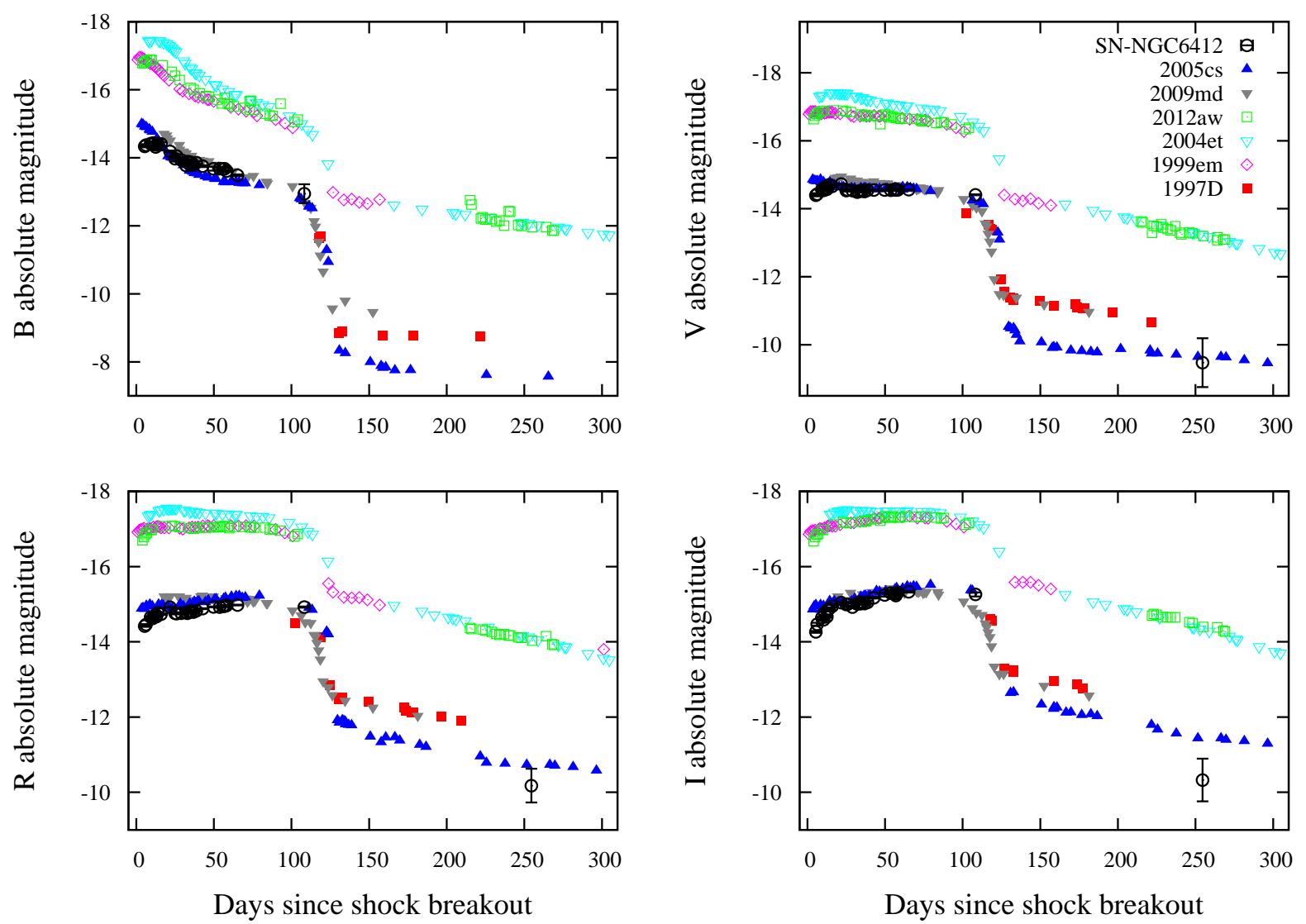

Figure 1. De-reddened absolute BVRI light curves of SN-NGC6412 together with those of other Type II-P SNe. Parameters for calculating the absolute light curves are taken from Table 2. The similarity between SN-NGC6412 and the low-luminosity Type II-P events (SN 1997D, 2005cs, 2009md) is apparent. Note: most of the time, the error bars are smaller than the point size.

2001) which is the prototype of low luminosity subclass. Unfortunately the light curve of SN 1997D is very sparsely sampled. SN $2009 \mathrm{md}$ is also a subluminous SN, which had a low mass progenitor similar to SN 2005cs (Fraser et al. 2011).

SN 1999em (Leonard et al. 2002), SN 2004et (Sahu et al. 2006) and SN 2012aw (Bose et al. 2013) on the other hand, are well-observed, normal II-P supernovae. They serve as a control group for the modeling program used below (Section 8).

\section{SPECTROSCOPY}

There is only a single observed spectrum available for SN-NGC6412 in the literature (Tomasella et al. 2015) taken one day after the discovery (57214 MJD). It is shown in Fig. 2 top left panel. The spectrum is contaminated by telluric lines as well as emission features from the host galaxy. Before further analysis, the following host galaxy features were removed from the spectrum: H г $\lambda \lambda 4341,4861,6563, \mathrm{~N}$ i $\lambda \lambda 6548,6583$, О г $\lambda \lambda 3727$, O III $\lambda \lambda$ 4959, 5007, Mg Iі $\lambda \lambda$ 2798, S Iі $\lambda \lambda 6717,6731$, Ca H\&K.

We utilized the Syn++ modeling code
(Thomas et al. 2011) to fit the observed spectrum after correcting it for extinction and redshift. Syn++ is an advanced version of SYNOW which uses the Sobolev approximation (see Fisher et al. 1997) to calculate the $\mathrm{P}$ Cygni features formed by pure resonance scattering in a homologously expanding SN atmosphere.

The spectrum of SN-NGC6412 (taken on 2015-7-11, 57214 MJD) shows H, HeII, He I and NiII lines, which elements we expect in an early hydrogen-rich supernova atmosphere (see Hatano et al. 1999, Fig. 2a). The parameters used for the fitting are shown in Table 3 . The feature at $4578 \AA$, can be either He II or high velocity H $\left(23000 \mathrm{~km} \mathrm{~s}^{-1}\right)$. However a high velocity $\mathrm{H}$ would imply a remarkable absorption around $6095 \AA$, which is not the case (Fig. 2). That is why we adopted He II. This is also consistent with the temperature. The formation of the $\mathrm{He}$ II line requires high temperature. The temperature is $19000 \pm 3000 \mathrm{~K}$, while the photospheric velocity of the best-fit model is $7000 \mathrm{~km} \mathrm{~s}^{-1}$, which implies a very early stage. The spectrum comparison made with the SNID (Supernova Identification, Blondin \& Tonry 2007) reveals the spectrum of SN 2005cs, taken on 2005-07-01, 2005 (4 days after the shock breakout), being the most similar one. However, SN 2005cs lacks the strong $\lambda 4578$ feature that is apparent is SN-NGC6412 (attributed to 


\begin{tabular}{|c|c|c|c|c|}
\hline SN & $\begin{array}{c}\text { Explosion } \\
\text { epoch [MJD] }\end{array}$ & $\begin{array}{c}\text { Distance } \\
{[\mathrm{Mpc}]}\end{array}$ & $\begin{array}{c}\mathrm{E}(\mathrm{B}-\mathrm{V}) \\
{[\mathrm{mag}]}\end{array}$ & Reference \\
\hline 1997D & $50361.0^{*}$ & 13.4 & 0 & Benetti et al. 2001; Spiro et al. 2014 \\
\hline $1999 \mathrm{em}$ & 51480.4 & 11.7 & 0.1 & Leonard et al. 2002; Leonard et al. 2003 \\
\hline 2004et & 53270.0 & 5.6 & 0.41 & Sahu et al. 2006 \\
\hline $2005 \mathrm{cs}$ & 53549.0 & 7.1 & 0.05 & Pastorello et al. 2009; Takats \& Vinko 2006 \\
\hline $2009 \mathrm{md}$ & 55162.0 & 21.28 & 0.1 & Fraser et al. 2011 \\
\hline 2012aw & 56002.1 & 9.9 & 0.074 & Bose et al. 2013 \\
\hline SN- & & & & \\
\hline NGC6412 & 57210.0 & 17.85 & 0.035 & This work \\
\hline
\end{tabular}

Table 2. Adopted parameters. * SN 1997D explosion epoch is uncertain. We adopted the value given by (Spiro et al. 2014) with extended plateau range. Explosion epoch discuss is can be found in Section 6, distance discuss in Section 4, and extinction estimation is in Section 3.

HeII, see above). At that epoch the photospheric velocity of SN $2005 \mathrm{cs}$ was $6950 \mathrm{~km} \mathrm{~s}^{-1}$, while its temperature was $13350 \mathrm{~K}$ (Dessart et al. 2008) As we can see the expansion velocity is very similar to the SN-NGC6412.

The difficulties of determining the continuum of a noisy spectrum render any measurement of equivalent widths very uncertain. Nevertheless, we performed such a procedure for the $\mathrm{P}$ Cygni profile of the $\mathrm{H} \alpha$ line, in order to have some rough quantities for comparison with other supernovae (Fig. 2, bottom two panels). The equivalent widths are 5 for the absorption part and 37.2 for the emission part, giving an absorption/emission ratio $a / e=0.13$. For comparison, SN2005cs has the corresponding values of $9.8 \pm 1$ and $79.5 \pm 1.1$ for absorption and emission respectively, and a ratio $a / e=0.12$. The $a / e$ is similar for the two, however for SN-NGC6412 it may be higher due to the uncertainty of the continuum. The SNe start with low $a / e$ which then increase over time as the temperature drops (see Gutierrez et al. 2017). SN-NGC6412 has a smaller equivalent widths than the SN 2005cs. This is because of the higher temperature.

The upper limit of the velocities for SN-NGC6412 reaches $30000 \mathrm{~km} \mathrm{~s}^{-1}$ (see Table 3), resulting in a longer shallow absorption. However Syn++ fit shows that this feature is the part of the outer atmosphere, and not a high velocity Hydrogen feature.

\section{THE EVOLUTION OF TEMPERATURE AND RADIUS AT THE PHOTOSPHERE}

Using the photometry of SN-NGC6412, we determined the temperature $(T)$ and radius $(R)$ of the photosphere of the SN. The spectral energy distribution (SED) is modeled as a diluted blackbody radiation, using the following formula:

$$
F_{\lambda}=(R / d)^{2} \cdot \xi^{2}(T) \cdot \pi \cdot \mathrm{B}(\lambda, T) \cdot 10^{-0.4 \cdot A_{\lambda}}
$$

where $F_{\lambda}$ is the flux, $R$ is the radius, $d$ is the distance, $\lambda$ is the wavelength, $\mathrm{B}$ is the Planck function, $A$ is the extinction, and $\xi(T)$ is the dilution factor (Eastman et al. 1996; Hamuy et al. 2001; Dessart \& Hillier 2005) that corrects the flux of a pure blackbody to the one formed in a strongly scattering $\mathrm{SN}$ atmosphere. $\theta=R / d$ is the angular radius of the photosphere.

The fitting of Equation (2) was also done to all of the other $\mathrm{SNe}$ used for comparison. Fig. 3 show the $T$ and $R$ values obtained in this way. The B band flux was not included in the blackbody fitting, because the blackbody flux in the blue and UV region significantly differs from the observed fluxes. However the photospheric temperature and radius values for the reference $\mathrm{SNe}$ in the literature were computed including the B flux in the fitting. This explains the slightly different values obtained for the same $\mathrm{SNe}$, as seen in Fig. 3. Nevertheless all of the SNe seem to evolve in a similar way: the initial high temperature decreases very quickly, and at the end of the plateau phase it becomes quasi-constant around $7000 \mathrm{~K}$, which is close to the recombination temperature of the $\mathrm{H}$. The radius shows a linear expansion at the beginning, but after a while it also becomes quasi-constant, and dropping down at the end of the plateau. The temperature of SN-NGC6412 evolves similar to the other $\mathrm{SNe}$, except for the first point which gives $20000 \mathrm{~K}$. This suggest a very early epoch. SN-NGC6412 and SN 2005cs have significantly lower photospheric radii, suggesting low photospheric velocities. Also, this might be the reason why they are very dim.

The time of shock breakout $\left(t_{0}\right)$ is an important required parameter for the modeling of the LC. Naturally $t_{0}$ must be earlier than the discovery epoch ( 57213 MJD). Epochs significantly earlier than the discovery are unlikely because the temperature on the first observed epoch was quite high (20000 K see Fig. 3), implying that it is a very early epoch, even down to only one day. The mean of these two value is 57210.5. Also, if $t_{0}=$ 5210 MJD, the photosphere radius and the bolometric LC of SN-NGC6412 and SN 2005cs become very similar (see Section 7). Therefore we adopt $t_{0}=57210 \pm 2 \mathrm{MJD}$ as the epoch for the shock breakout. Fortunately the uncertainty is quite low, making it adequate for the modeling. 

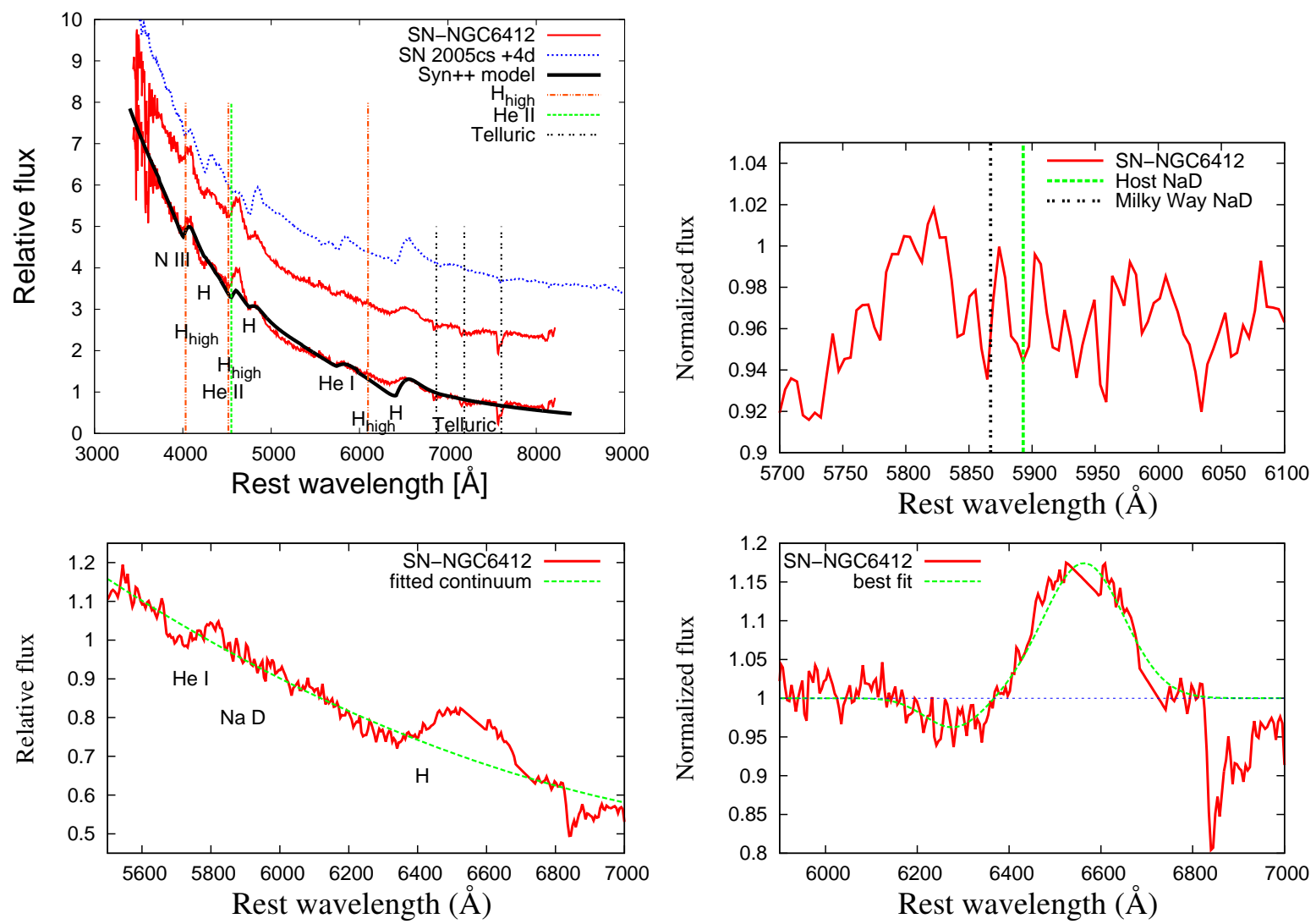

Figure 2. Upper left: The spectrum of SN-NGC6412 corrected for redshift, the best fitting Syn++ model, and the spectrum of SN 2005cs (shifted vertically for easy visual comparison) The feature at $4578 \AA$ can be fitted with high velocity $\mathrm{H}$ (23000 km $\mathrm{s}^{-1}$ ) and with H II. See text. Upper right: The Na D line in SN-NGC6412 spectrum. Bottom left: The region around the $\boldsymbol{H}_{\boldsymbol{\alpha}}$ in SN-NGC6412 spectrum. Bottom right: The P Cygni profile of the $\boldsymbol{H}_{\boldsymbol{\alpha}}$, and the fit used to calculate the equivalent width, see text.

\begin{tabular}{|c|c|c|c|c|c|}
\hline Parameter & Photosphere & $\mathrm{H}$ & He II & He I & N III \\
\hline Velocity $\left[\mathrm{km} \mathrm{s}^{-1}\right]$ & 7000 & 7000 & 9000 & 7000 & 7000 \\
\hline MAX Velocity $\left[\mathrm{km} \mathrm{s}^{-1}\right]$ & 30000 & 30000 & 30000 & 30000 & 30000 \\
\hline Temperature $[\mathrm{K}]$ & 19000 & 19000 & 19000 & 19000 & 19000 \\
\hline $\log (\tau)$ & - & -0.4 & -1.2 & -1.5 & -1.3 \\
\hline $\operatorname{aux}\left[\mathrm{km} \mathrm{s}^{-1}\right]$ & - & 8000 & 8000 & 8000 & 8000 \\
\hline
\end{tabular}

Table 3. Parameters used to fit SN-NGC6412 spectrum with Syn++.

\section{BOLOMETRIC LIGHT CURVE}

The modeling code requires the bolometric light curve as an input. Unfortunately, there are only optical data available. To handle this we have to make estimations for the regions not covered by the observations.

The total flux in the infrared (IR) region, covering wavelengths beyond the I band, was calculated as the integral of the fitted diluted black body radiation. The diluted black body is only fitted to bands that are red-ward from the B-band, because the blue part of the spectrum significantly differs from that of black body radiation.

We tested the effect of the inclusion/omission of the B-band flux into/from the blackbody fitting in the following way. For those SNe that had JHK data available, the flux integral in the near-IR regime was also calculated via direct integration of the $J H K$ fluxes serving as a control. In addition, the IR flux was also estimated by fitting a Rayleigh-Jeans (RJ) tail to the I-band flux and integrating it between the I-band and infinity. These results (the direct integration and the RJ approximation) were then compared to the integral of the fitted blackbody, which was computed with and without the B-band flux. It turned out that omitting the B-band flux from the blackbody fitting (i.e. fitting only to the $V R I$ bands) gives much closer values to the result from the direct integration, far better than the RJ approximation, at least during the photospheric phase. Fig. 4 shows the results of the testing. The green-coloured open symbols depict 

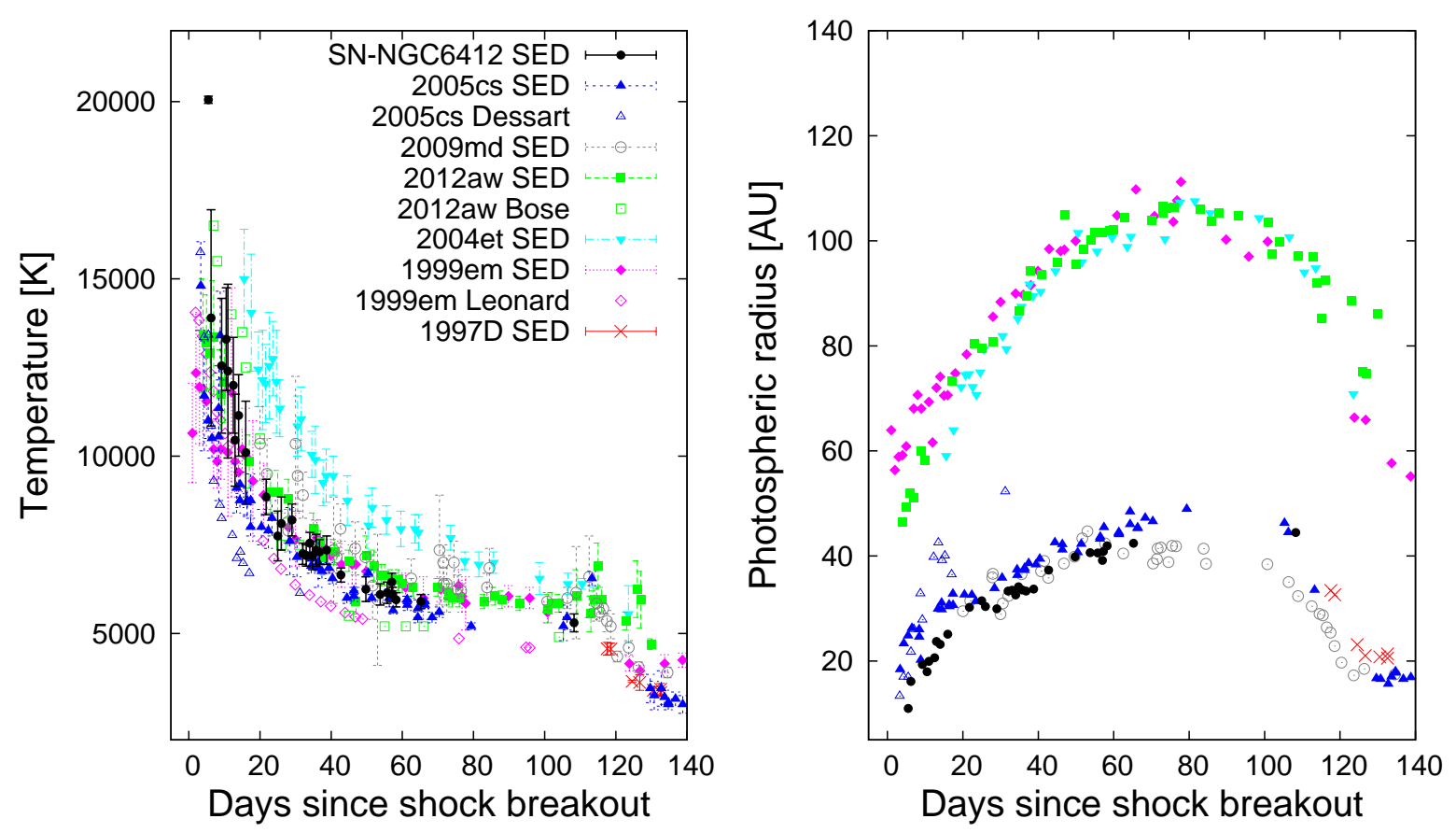

Figure 3. Evolution of the photospheric temperatures (left) and radii (right) of the discussed SNe. Filled symbols represents the results from the SED fitting (see text), while open symbols corresponds to values collected from literature. The first point of SN-NGC6412 gives $~ 20000 \mathrm{~K}$ which is consistent with the value comes from the spectrum fit (19000 K)

the actual integration using UV, optical and IR data, serving as a control. The plot only includes points with either UV or IR data available beside the optical data (SN 2012aw only had optical at the tail phase, thats is why it is missing from the plot). The filled black circles show the estimation excluding the B band, the red filled triangles show the estimation with $\mathrm{B}$ band, and the blue filled upside down triangles show the RJ approximation.

This method does not work in the nebular phase, because the blackbody assumption breaks down after the plateau as the ejecta becomes more and more transparent. Thus, after the plateau phase we applied only the direct integration of the near-IR fluxes, except for SN 1999em, where we added the integral of a RayleighJeans tail fitted to the observed I-band fluxes. The same technique was applied to the single epoch $B V R I$ fluxes of SN-NGC6412 taken after the end of the plateau phase.

The flux in the ultraviolet (UV) region, encompassing wavelengths shorter than band $\mathrm{B}$, was extrapolated to $2000 \AA$ from the $\mathrm{B}$ and $\mathrm{V}$ bands, and assumed zero for even shorter wavelengths (Lyman et al. 2014). For the optical region we integrated the observed and de-reddened fluxes over the spectral bands using the trapezoidal rule. Adopted distance and extinction values are shown in Table 2 .

The data for the comparison $\mathrm{SNe}$ were collected from the following sources. SN 2005cs: optical and NIR: Pastorello et al. (2009); UV: Brown et al. (2007). SN 2012aw: optical: Bose et al. ( 2013); Dall'ora et al. (2014); NIR (dates preceding the nebular phase): Dall'ora et al. (2014); UV: Bayless et al. (2013).
SN 2004et: optical: Sahu et al. ( 2006); NIR (after the tail): Maguire et al. ( 2010). SN 1999em only has existing optical data: Leonard et al. ( 2002). SN 2009md: optical and NIR (after the tail): Fraser et al. ( 2011).

The errors for the bolometric fluxes were calculated as follows. The optical and UV flux errors were estimated from the error propagation while integrating the flux with the trapezoidal rule. The IR flux error comes from the uncertainty of the temperature of the fitted blackbody. However, this does not include the uncertainty due to the validity of the applied blackbody model. Thus, in order to include this kind of uncertainty in the total error budget, we added a fixed percent of the UV and IR flux to the formal errors mentioned above. For the IR the percentage depends on the goodness of fit of the diluted blackbody: we define it as the flux ratio of the direct integral of the optical fluxes and the integral of the blackbody in the optical region. For UV it was fixed as $50 \%$. With this value, the uncertainty of the bolometric magnitude is $0.3 \mathrm{mag}$ at the beginning of the $\mathrm{LC}$. This is the approximate error in the $\mathrm{BC}_{B}-(B-I)$ relation around $B-I=0$ (see Fig. 5).

Gaps in the optical band were filled in by linear interpolation. Bolometric fluxes were generated only for measurements involving at least 3 optical bands.

To check the correctness of this method, we used an empirical correlation found by Lyman et al. ( 2014) between the bolometric correction of the $B$ band $\left(\mathrm{BC}_{B}=\right.$ bolometric magnitude minus B-band magnitude) and the color index $B-I$. They modeled the correlation 
by fitting a second order polynomial to it. We plotted $\mathrm{BC}_{B}=$ vs $B-I$ for every $\mathrm{SN}$ in Fig. 5 . The plot shows that SN 2005cs and the other SNe closely follow this model, and SN-NGC6412 also closely follows this if the extrapolation is limited to $\lambda \geq 2800 \AA$, instead of $2000 \AA$. This means that the two methods (integral, and the Lyman model) give the same result.

However the very early UV flux is still highly unreliable and the Lyman model does not fit it well. SN 2005cs and SN 2012aw have existing UV observations and they were used to test the UV region. We fitted the deviation from the Lyman model with a straight line:

$$
\mathrm{BC}_{B}=0.51 \cdot(B-I)-0.48 \quad \text { if }(B-I)<0.54
$$

The other SNe with the extrapolated UV flux show the same deviation from the Lyman model at the beginning, except for SN-NGC6412. The bolometric light curves corrected in this way are shown in Fig. 6. In the literature, RJ approximation was used for SN 1999em instead of blackbody fitting (Elmhamdi et al. 2003). This approximation is adequate for the nebular phase, but not for the photometric phase, as can be seen in Fig. 4. Thus, we used the better BB fitting method (see Fig. 5, and Fig. 6).

Note that in Fig. 5 SN 2005cs, and to some extent SN 1997D and 2009md deviate from the Lyman model in the high $B-I$ values. The same figure also shows the development in time of the $B-I$ colour. At first all $\mathrm{SNe}$ start with $B-I \sim 0$, meaning that the ejecta is blue in colour. As the temperature drops over time (Fig. 3), the $B-I$ colour increases. At the end of the plateau it suddenly rises. The LL SNe have an even higher rise of $B-I$ than regular Type II-P SNe, reaching $B-I=4.5$ mag. This is a unique feature of LL SNe. The sample made by Lyman et al. ( 2014) was only fitted till about $B-I=\sim 3$ mag. This model at $B-I=4.5 \mathrm{mag}$ is not entirely valid, however, it is more or less still adequate.

\section{BOLOMETRIC LIGHT CURVE FITTING, AND THE MODELING CODE}

We used an upgraded version of the LC2.2 semianalytic light curve code (see Nagy \& Vinko 2016) to fit the quasi-bolometric light curve. This code is based on a model that was originally described by Arnett \& Fu (1989) and later extended by Popov (1993); Blinnikov \& Popov ( 1993) and Nagy et al. ( 2014). A wide variety of SN light curves can be modeled with this code, depending on the choice of the initial parameters, such as the ejected mass $\left(M_{\mathrm{ej}}\right)$, the initial radius of the progenitor $\left(R_{0}\right)$, the total explosion energy $\left(E_{0}\right)$, and the mass of the synthesized ${ }^{56} \mathrm{Ni}\left(M_{\mathrm{Ni}}\right)$ which at later phases directly determines the emitted flux. The model assumes a homologously expanding and spherically symmetric SN ejecta having a uniform density core and an exponentially decreasing density profile in the outer layers. The diffusion approximation was used for the radiation transport. The recombination causing the rapid change of the effective opacity in the enve-
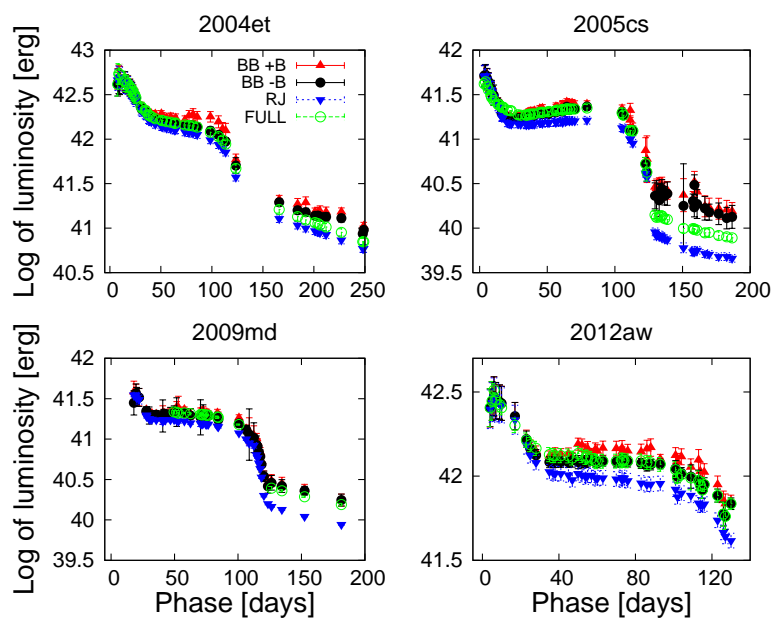

Figure 4. Comparing the methods to calculate bolometric flux. The Figure only show those SNe which has both UV and IR data available beside the optical data. BB: the fitted blackbody, which was computed with $(+\mathrm{B})$ and without (-B) including the B-band flux. RJ: Rayleigh-Jeans approximation. FULL: Direct integral using JHK and Swift (where available) fluxes. Optical regime calculated with direct integral in any case. UV regime calculated with interpolation (see text) where Swift flux was unavailable.

lope is taken into account in a very simple form (see Arnett \& Fu 1989, Eq. (A8)).

The LC2.2 code is only a modeling tool, thus it does not contain any routine for numerically fitting the output model to the observed light curves. Thus we added a Markov Chain Monte Carlo (MCMC) method using the Metropolis-Hastings Algorithm with Gibbs sampler (Metropolis et al. 1953; Hastings 1970; Gilks et al. 1996), to find the best fits to the bolometric light curve (which are selected by the least squares method, $\chi^{2}=\Sigma_{i}^{N}\left(\frac{M_{i}-D_{i}}{\sigma_{i}}\right)^{2}$, where M: model, D: data, $\sigma$ : measurement errors), explore the parameter space, and investigate correlations between the parameters.

The MCMC program samples the joint posterior probability distribution of the parameters, given suitable a priori probability distributions for each parameter and the goodness of fit, $\chi^{2}$, which establishes the likelihood as $\mathcal{L}=\exp \left(-\chi^{2} / 2\right)$ (We assume Gaussian errors as usual).

The accepted maximum and minimum parameter regions are based on Hamuy ( 2003), however the range has been extended to be more general.

Because of the direct sampling of the parameter space, any correlation between the parameters can also be readily observed. There are two known main parameter correlations (Arnett \& Fu 1989, Nagy et al. 2014): between $M_{\mathrm{ej}}$ and $E_{\mathrm{kin}}$, and between $R_{0}$ and $E_{\mathrm{th}}$. The opacity $(\kappa)$, and the exponent of the power-law density profile (of mass) $(s)$ may also be sampled, which are also known to have correlations (Nagy \& Vinko 2016)). There are other parameters not entering into the sampling process, i.e. they are not fitted. These include the ionization temperature $\left(T_{\mathrm{ion}}\right)$, the date of explosion $\left(t_{0}\right)$, and the distance $(d), t_{0}$ and $d$ can be determined inde- 

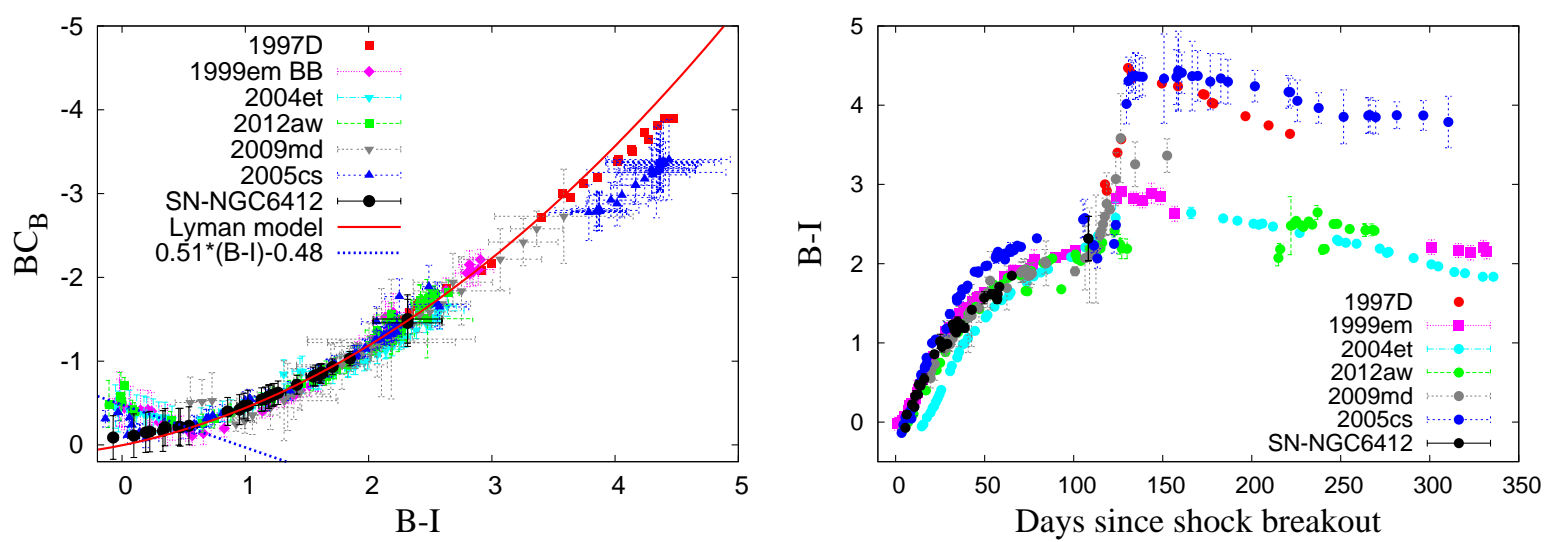

Figure 5. Left: The $\mathrm{BC}_{B}-(B-I)$ relation for the SNe in this study: For $B-I<0.8$ we apply the prediction from the linear fitting (blue dotted line) instead of the fitted polynomial (red curve). Right: the colour (B-I) development over time.

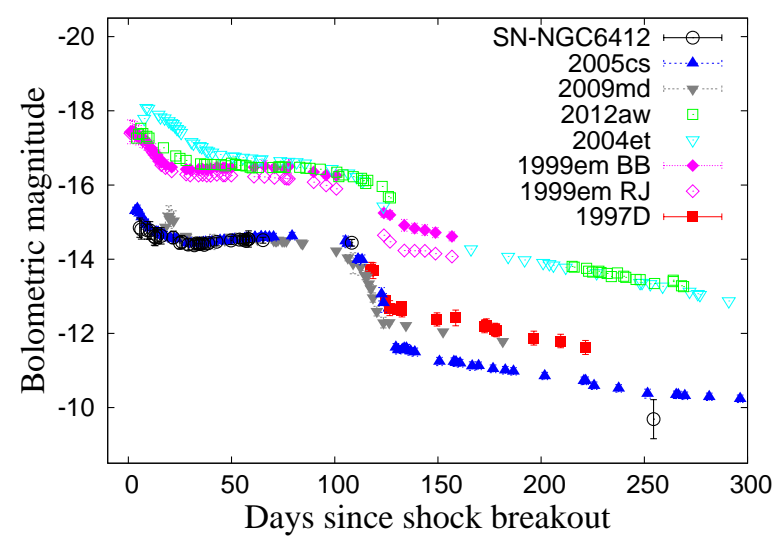

Figure 6. Bolometric light curves of the SNe.

pendently, and $T_{\text {ion }}=5500 \mathrm{~K}$ was adopted as the ionization temperature of the hydrogen. (Note: if $t_{0}$ and $d$ are not well known, the algorithm, after including them into the fitting process, is also able to determine their uncertainties.) Using constant density model $s=0$ gives a good agreement with the hydrodynamical models, so $s=0$ was adopted. The opacity $\kappa$ also correlates with $E_{\text {kin }}$ and $M_{\mathrm{ej}}$. Because of this we have also sampled $\kappa$ between $\kappa=0.3 \mathrm{~cm}^{2} \mathrm{~g}^{-1}$ and $\kappa=0.2 \mathrm{~cm}^{2} \mathrm{~g}^{-1}$ (with uniform prior: $\kappa=[0.2: 0.3] \mathrm{cm}^{2} \mathrm{~g}^{-1}$ ), These are the average opacities calculated by the public code SNEC (Morozova et al. 2015; see Nagy \& Vinko 2016 for details).

Because the light curve sampling is not very dense, to say the least, we had to take some measures to ensure a correct fit of the model on the fluxes. The weights of the last two points have been increased (their uncertainties decreased 10 times), to ensure that the these important points are properly fitted (in other words, the model goes through or close to these important points).

Also, the first 30 days after the explosion are not fitted, because the bolometric fluxes are uncertain in that regime, due to a lack of UV flux measurements. Luckily this phase of the light curve is caused by the ejected outer shell and not the core, and this shell is independent from the core as it has different physical parameters, for example larger radius, so it must be fitted separately. (see Nagy \& Vinko 2016 for more information).

The error of $t_{0}$ is very low so it does not affect the other parameters significantly. The tail of SN-NGC6412 seems to be 4 magnitude dimmer than at the end of the plateau, just like in the case of SN $2005 \mathrm{cs}$. This means $\sim 35$ times lower flux, so it adds a very small portion of energy to the luminosity.

\section{THE MISSING PLATEAU ENDPOINT AND ITS IMPLICATIONS}

The code was applied to the data of SN-NGC6412 and to the other comparison/reference SNe. The posterior distributions and the best fits of SN-NGC6412 are shown in (Fig. 7) $\left(d=17.85 \mathrm{Mpc}, \kappa=[0.2: 0.3] \mathrm{cm}^{2}\right.$ $\left.\mathrm{g}^{-1}\right)$. The posterior distributions of the reference objects SN 1999em, SN 2004et, SN 2005cs and SN 2012aw are shown in the Appendix. The detailed discussion of the results is given in Section 10 .

The unfortunate lack of an observed plateau endpoint is a serious issue. The light-curves of SN 2005cs and SN-NGC6412 are running closely together up to the last plateau observation (Fig. 6). Luckily the MCMC algorithm samples the whole parameter space and searches for all possible solutions with different plateau endpoints. The shape of the observed data constrains the endpoint position. In our case the absence of endpoint observation gives rise to a secondary maximum (a case of multi-modal distribution) in the parameter space, a solution with larger $M_{\mathrm{ej}}$. A comparison of the model light-curves of the two maxima, which can be seen on (Fig. 7), clearly shows that the solution corresponding to the second maximum is far worse. The endpoint of the plateau is also too far away. A comparison with other LL SNe also reveals that such a late endpoint is very unlikely, see also Table 4 showing the plateau end epoch and luminosity at the 50th day. The plateau end 
epoch is between day 100 and 130, while the luminosity difference is almost a factor of 10 .

The spectrum of SN-NGC6412 is also similar to that of SN 2005cs at the similar epoch, having nearly the same early photospheric velocity, which suggests that they have similar expansion velocities at their outmost layers. The development of the photospheric radius (Fig. 3, right panel) is also very similar. So we repeated the fitting while including also the expansion velocity with a Gaussian prior. We assume a homologously expanding spherical symmetric ejecta. The expansion velocity is a calculated parameter, which stands for the velocity of the outermost layer of the SN ejecta (see Nagy \& Vinko 2016), and assumed to be constant. As the photosphere propagates inward the remnant, it will be located in a different radius resulting different photosperic velocities. The expansion velocity for SN-NGC6412 was determined from the SN 2005cs fit but with larger uncertainty: $3500 \mathrm{~km} \mathrm{~s}^{-1}$ mean and $2 \sigma=500 \mathrm{~km} \mathrm{~s}^{-1}$ (see Appendix for the exact values for SN 2005cs). After repeating the fitting with these modifications, the second maximum vanished and only the better one remained, as shown in Fig. 7. Note even with this, the uncertainty of the plateau end is still rather high ( $\sim 20$ day), however this uncertainty is included in the fit and the final results.

\section{RESULTS}

Fig. 7 shows that our results are consistent with Arnett \& Fu ( 1989) and Nagy et al. ( 2014), regarding the correlations between the parameters.

Due to the correlation between $R_{0}$ and $E_{\mathrm{th}}$, they cannot determined separately, only their product, $R_{0}$. $E_{\mathrm{th}}$, can be derived, and should be used as an independent parameter in subsequent analyses, instead of $R_{0}$ and $E_{\mathrm{th}}$.

For $M_{\mathrm{ej}}$ and $E_{\mathrm{kin}}$, their correlation shows a parabolic trend (in logarithm). This is a more complex correlation than we previously thought. We can not make an independent parameter from $M_{\mathrm{ej}}$ and $E_{\mathrm{kin}}$ because of this complex correlation trend. They are however, less significantly correlated (Fig. 7, left panel) and we can determine them separately quite well.

Other correlations between the main parameters have not been found to be significant. This is shown in Fig. 8 for SN-NGC6412 with added velocity prior.

Nagy \& Vinko ( 2016) showed that the opacity $(\kappa)$ also correlates with $M_{\mathrm{ej}}$. We also sampled the opacity $(\kappa)$ for additional correlations. A higher opacity increases both the velocity $(v)$ and $R_{0} \cdot E_{\mathrm{th}}$, but $M_{\mathrm{ej}}$ is the most affected. Increasing $\kappa$ substantially decreases $M_{\mathrm{ej}}$, while $E_{\text {kin }}$ is also changing, although less significantly; (Fig. 9). This may cause a higher $M_{\mathrm{ej}}$ while $E_{\text {kin }}$ remains about the same. This may be the cause for higher mass estimations from hydrocodes, while other methods imply lower masses (see the Appendix) However care should be taken with the interpretation, as our model uses constant opacities, which is an approximation.

Nevertheless these correlations are not so significant and only increase the uncertainties of the parameters slightly.

Increasing the value of the power-law density profile exponent $(s)$ significantly decreases $M_{\mathrm{ej}}$ and also decreases $E_{\text {kin }}$, although less significantly, while $R_{0} \cdot E_{\text {th }}$ increases. This is shown in Fig. 10. These are significant correlations, so we adopt the $s=0$ value, because it gives the values consistent with the literature and the hydrocodes. Larger power-law exponents give unrealistic results, like very low mass and too high velocities. This confirms Nagy \& Vinko ( 2016) where this statement was also tested. Correlation between $s$ and $\kappa$ have not been found in this parameter region.

Sampling $t_{0}$ and $d$ may also be possible, however doing so gives high uncertainties. Uncertainties in the date of plateau end and $t_{0}$ mostly increase the uncertainty of $M_{\mathrm{ej}}$, but other parameters also become more uncertain, although less significantly. Clearly it is more meaningful to have them determined independently.

The best values and the uncertainties of the fitted parameters for SN-NGC6412 are shown in Table 5. The mean values, uncertainties and comparison values from the literature for the other SNe are shown in Tables 6-9 in the Appendix (for SN 1999em, SN 2004et, SN 2005cs, and SN 2012aw respectively).

For SN-NGC6412 the fitting gives $R_{0}=$ $20-220 \cdot 10^{11} \mathrm{~cm}=28-315 R_{\odot}, M_{\mathrm{ej}}=8.5-11.5 M_{\odot}$, the energies $0.5-0.8$ foe, $v_{\text {exp }}=3300 \mathrm{~km} \mathrm{~s}^{-1}$. The initial nickel mass of SN-NGC6412 was $1.55_{-0.70}^{+0.75} \cdot 10^{-3} M_{\odot}$. These are very similar to the values of SN $2005 \mathrm{cs}$. Parameter comparison of the SNe is shown in (Fig. 11). These results suggest that the progenitor of $\mathrm{SN}$ NGC6412 had a moderate mass, and rather small radius, low velocities and energies along with very low nickel mass, similar to other LL SNe (Pastorello et al. 2004; Spiro et al. 2014). Hamuy (2003) showed correlation between energy and the nickel mass: larger nickel mass implies larger energy, which is consistent with our findings.

\section{SUMMARY}

We have made a photometric monitoring of SNNGC6412 with two telescopes. It turned out that SN-NGC6412 is a sub-luminous type II-P SN with low nickel mass very similar to the SN 2005cs.

There is only one spectrum of SN-NGC6412 available by Tomasella et al. ( 2015). Fitting the spectrum with Syn++ reveals the presence of a strong HeII line, but quite weak $\mathrm{H}$ lines and low photospheric velocity. He I and N III lines also present in the spectrum. The early photospheric velocity and the expansion velocity are also very similar to SN $2005 \mathrm{cs}$.

We fitted diluted blackbody radiation to the light curves in different photometric bands throughout the observed phases. The inferred photometric radii are smaller then those of regular II-P SNe, which also 

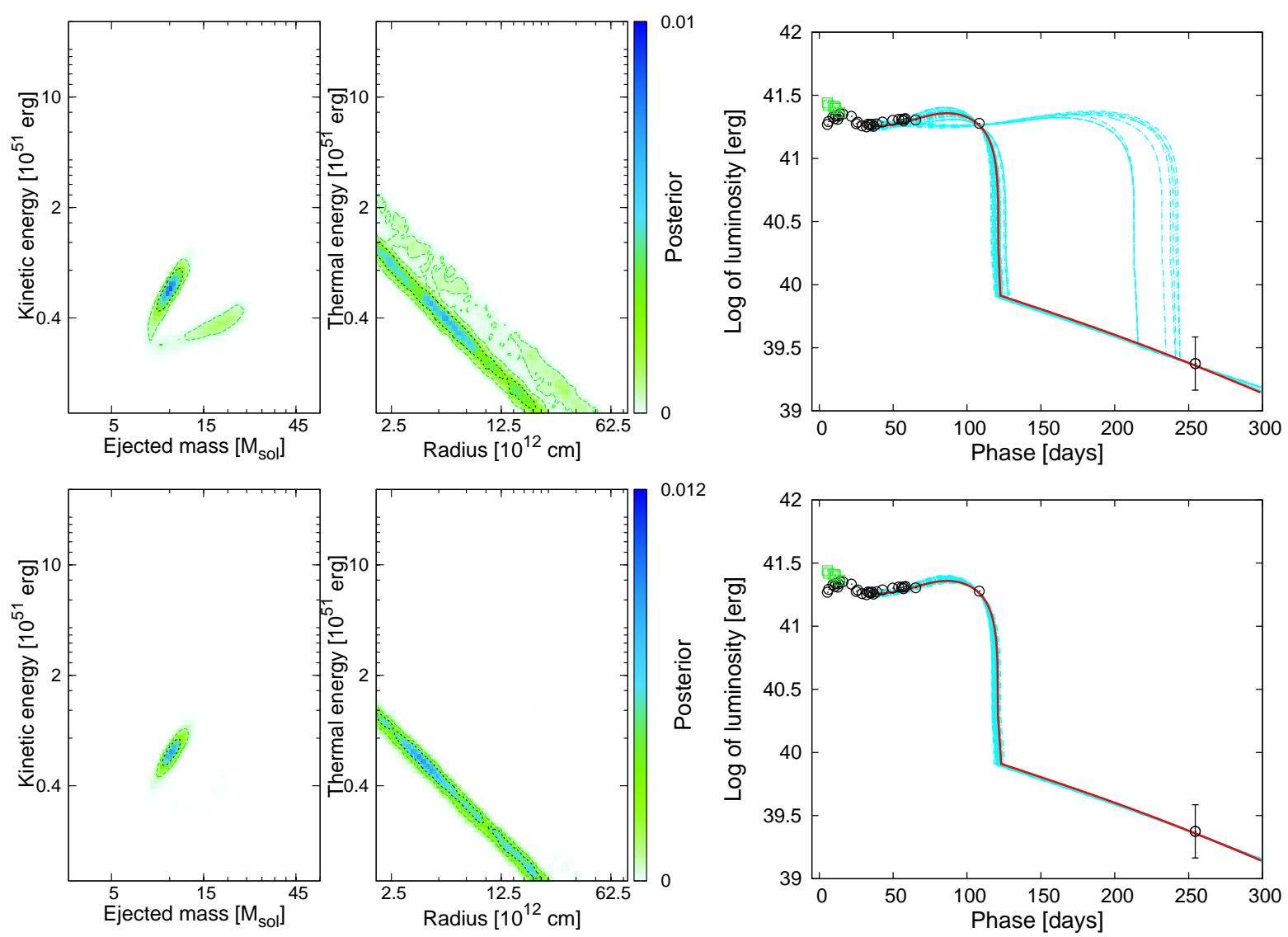

Figure 7. Upper left: probability distributions of SN-NGC6412 joint parameters, $d=17.85 \mathrm{Mpc}, s=0, \kappa=[0.2: 0.3] \mathrm{cm}^{2}$ $\mathrm{g}^{-1}$. The contours show the confidence interval of $67 \%$ and $95 \%$. Note that there is a second (multi-modal) pole. Upper right: SN-NGC6412 accepted fits (within 95\% probability contour) including the second (multi-modal) pole. The red line is the best fit. $d=17.85 \mathrm{Mpc}, s=0, \kappa=[0.2: 0.3] \mathrm{cm}^{2} \mathrm{~g}^{-1}$. The green symbols shows the added UV from the $B C_{B}(B-I)$ relation (Fig. 5). The second (multi-modal) pole fits have the end of the plateau too far, over +200 day, which is unrealistic. Bottom left: The probability distributions with given velocity prior. The second (multi-modal) pole vanished. Bottom right: SN-NGC6412 accepted fits with added velocity priori. Note that the bad fits vanished.

\begin{tabular}{lcccc}
\hline Name & $\log \mathbf{L}\left[\mathbf{e r g ~ s}^{-1}\right]$ @ day $\mathbf{5 0}$ & Plateau end $[\mathbf{d}]$ & $\boldsymbol{M}_{\mathbf{N i}}\left[0.001 \boldsymbol{M}_{\odot}\right]$ & Ref. \\
\hline SN 1997D & - & 125 & $5 \pm 4$ & $2,3,4$ \\
SN 1999br & 40.60 & - & $2 \pm 1$ & 1 \\
SN 1999eu & - & $100-120$ & $1 \pm 1$ & 1,4 \\
SN 2001dc & 40.85 & 110 & $5 \pm 2$ & 1 \\
SN 2003Z & 40.90 & 120 & $5 \pm 3$ & 4 \\
SN 2005cs & 41.10 & 120 & $3 \pm 1$ & 5 \\
SN 2008bk & 41.25 & 130 & $7 \pm 1$ & 6,7 \\
SN 2008in & 41.35 & 105 & $12 \pm 5$ & 8 \\
SN 2009N & 41.50 & 110 & $20 \pm 4$ & 9 \\
SN 2009md & 41.05 & 115 & $4 \pm 1$ & 10 \\
SN 2010id & 40.75 & 120 & - & 11 \\
SN-NGC6412 & 41.10 & $110-130$ & $1.5 \pm 0.8$ & this work \\
\hline
\end{tabular}

Table 4. The LL SNe family features. Ref.: (1) Pastorello et al. ( 2004); (2) Turatto et al. ( 1998); (3) Benetti et al. ( 2001); (4) Spiro et al. ( 2014); (5) Pastorello et al. ( 2009); (6) Mattila et al. ( 2008); (7) Van Dyk et al. ( 2012); (8) Roy et al. ( 2011); (9) Takats et al. ( 2014); (10) Fraser et al. ( 2011); (11) Gal-Yam et al. ( 2011); 


\begin{tabular}{lcccc}
\hline Parameter & $2 \sigma$ & $1 \sigma$ & $2 \sigma$ & $1 \sigma$ \\
\hline$R_{0}\left[10^{11} \mathrm{~cm}\right]$ & $176_{-155}^{+65}$ & $176_{-152}^{+55}$ & $91_{-70}^{+119}$ & $91_{-61}^{+110}$ \\
$M_{\mathrm{ej}}\left[M_{\odot}\right]$ & $9.90_{-1.34}^{+1.22}$ & $9.90_{-0.68}^{+0.42}$ & $9.89_{-1.00}^{+2.10}$ & $9.89_{-0.31}^{+0.83}$ \\
$E_{\mathrm{kin}}\left[10^{51} \mathrm{erg}\right]$ & $0.67_{-0.19}^{+0.18}$ & $0.67_{-0.07}^{+0.06}$ & $0.65_{-0.18}^{+0.19}$ & $0.65_{-0.06}^{+0.08}$ \\
$E_{\mathrm{th}}\left[10^{51} \mathrm{erg}\right]$ & $0.15_{-0.05}^{+0.97}$ & $0.15_{-0.04}^{+0.83}$ & $0.28_{-0.17}^{+0.77}$ & $0.28_{-0.17}^{+0.70}$ \\
$E_{\mathrm{th}} R_{0}\left[10^{62} \mathrm{erg} \mathrm{cm}\right]$ & $26.3_{-3.1}^{+1.3}$ & $26.3_{-0.2}^{+0.2}$ & $25.6_{-5.1}^{+4.1}$ & $25.6_{-2.5}^{+1.0}$ \\
$v_{\mathrm{exp}}\left[\mathrm{km} \mathrm{s}^{-1}\right]$ & $3367_{-382}^{+181}$ & $3367_{-17}^{+17}$ & $3332_{-347}^{+116}$ & $3332_{-17}^{+17}$ \\
$M_{\mathrm{Ni}}\left[0.001 M_{\odot}\right]$ & $1.55_{-0.70}^{+0.75}$ & $1.55_{-0.70}^{+0.75}$ & $1.55_{-0.70}^{+0.75}$ & $1.55_{-0.70}^{+0.75}$ \\
$\kappa\left[\mathrm{cm}^{2} \mathrm{~g}^{-1}\right]$ & 0.3 & 0.3 & $0.2: 0.3$ & $0.2: 0.3$ \\
\hline
\end{tabular}

Table 5. SN-NGC6412 LC fit values. Power-law density profile exponent $s=0$. The $2 \sigma$ is for the $95 \%$ confidence interval, and $1 \sigma$ is for the $67 \%$. The first two column is values with fixed opacity $(\kappa)$, while in the second two column the $\kappa$ is sampled as well.

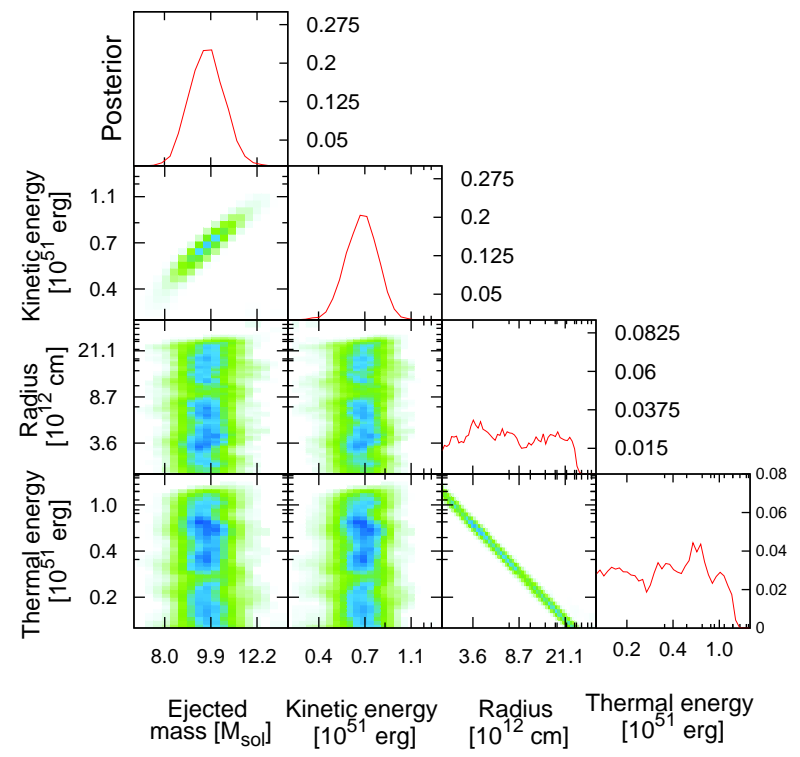

Figure 8. Correlations between the main parameters shown of SN-NGC6412 with added velocity prior. The color shows the probability distributions of the joint parameters. Here $s=0, \kappa=0.3 \mathrm{~cm}^{2} \mathrm{~g}^{-1}$ were chosen.
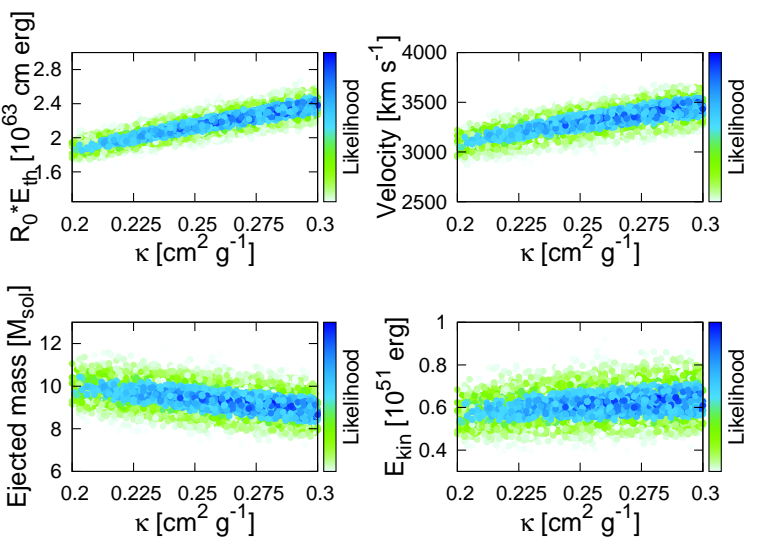

Figure 9. Opacity $(\boldsymbol{\kappa})$ correlations for SN 2005cs. Within this range, it does not affect the parameters significantly. $s=0$.
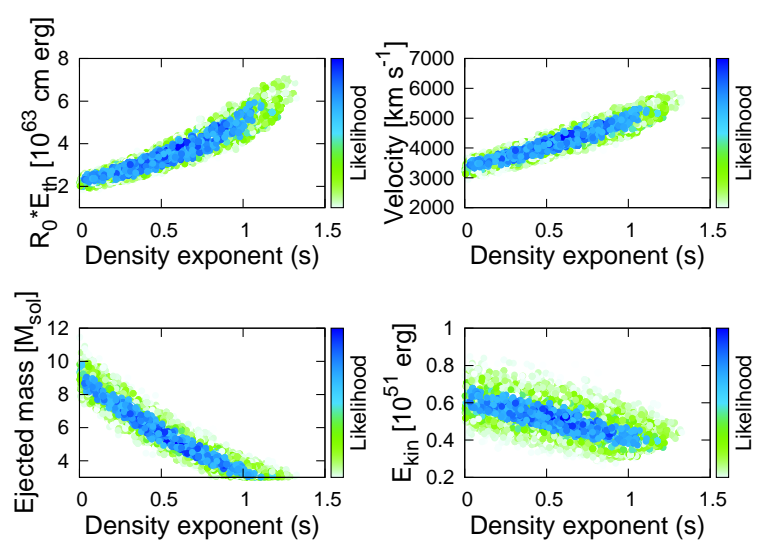

Figure 10. power-law density profile exponent correlations for SN 2005cs, $\kappa=[0.2: 0.3] \mathrm{cm}^{2} \mathrm{~g}^{-1}$. Larger power-law density profile exponent values gives unrealistic results e.g very low mass and too high velocities.

suggests low expansion velocities.

We modeled the bolometric light curve of SNNGC6124 with our new fitting code that uses the MCMC method to find the most probable fitting parameters together with their exact uncertainties, and examine the correlations between the parameters. With this we can reliably determinate the limits of our inferred parameters.

Besides the already known correlations between $M_{\text {ej }}$, $E_{\text {kin }}, R_{0}$ and $E_{\mathrm{th}}$, we find that the optical opacity $(\kappa)$ correlates with practically all other parameters, but the correlation is weak. $R_{0}$ and $E_{\mathrm{th}}$ cannot be separated, and only their product can be determined from the fitting. The correlation between $M_{\mathrm{ej}}, E_{\mathrm{kin}}$ (and $\kappa$ ) is weak, and both parameters can be determinated separately very well. The power-law density profile exponent $(s)$, however, shows significant correlations with the other parameters. We confirm that $s=0$ (constant density) results in the most realistic parameter values.

The most probable fitting parameters for SNNGC6412 with 0.95 level confidence errors are the following: initial radius $R_{0}=91_{-70}^{+119} \cdot 10^{11} \mathrm{~cm}$, ejecta mass 

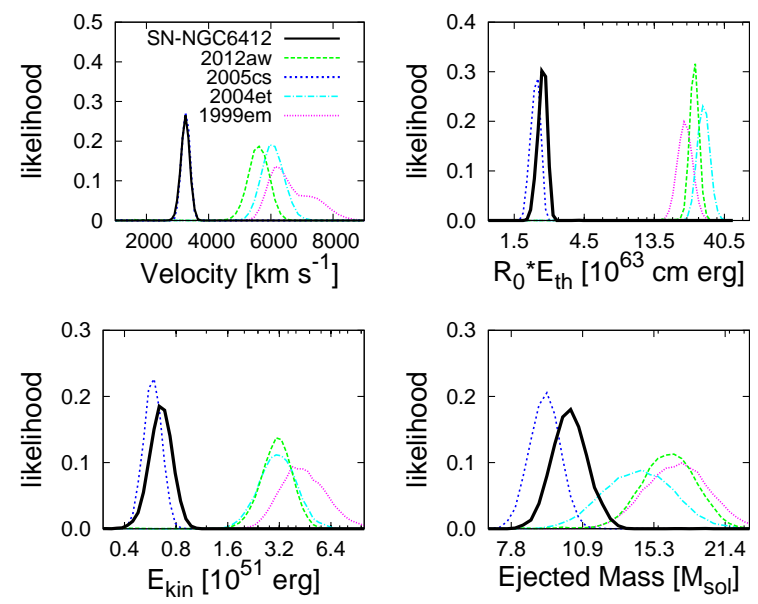

Figure 11. Parameter marginalization comparison, $\kappa=[0.2$ : $0.3] \mathrm{cm}^{2} \mathrm{~g}^{-1}, s=0$. (1999em: BB fit used, see text)

$M_{\mathrm{ej}}=9.89_{-1.00}^{+2.10} M_{\odot}$, kinetic energy $E_{\mathrm{kin}}=0.65_{-0.18}^{+0.19}$ foe, expansion velocity $v_{\exp }=3332_{-347}^{+216} \mathrm{~km} \mathrm{~s}^{-1}$. The initial nickel mass of SN-NGC6412 was $1.55_{-0.70}^{+0.75} \cdot 10^{-3} M_{\odot}$. These values are very similar to those of SN $2005 \mathrm{cs}$.

The inferred physical parameters of the ejecta suggest that SN-NGC6412 most probably arose from a moderate-mass progenitor just as SN 2005cs did. This may give further support to the hypothesis that most of such low luminosity Type II-P SNe are due to the core collapse of moderate-mass $\left(8-10 M_{\odot}\right)$ red supergiant (RSG) stars. They are good candidates for the electron capture SNe with O-Ne-Mg core. Obviously our present dataset is insufficient to decide whether SN-NGC6412 had such a core. The family of LL SNe is still mysterious. Further studies are recommended.

We also note that, despite the unfortunate fact that the brightness change of SN-NGC6412 has been not sampled adequately, which would give very high uncertainties, by correctly including independent observational evidence in the analysis, namely radial velocity as an a priori probability distribution, the parameters have become quite well constrained. This gives hope that the analysis of other similarly poorly sampled supernovae can also be attempted with the same methodology.

\section{ACKNOWLEDGMENTS}

This work is part of the project "Transient Astrophysical Objects" GINOP 2.3.2-15-2016-00033 of the National Research, Development and Innovation Office (NKFIH), Hungary, funded by the European Union.

L. M. was supported by the Premium Postdoctoral Research Program of the Hungarian Academy of Sciences. The research leading to these results has received funding from the LP2018-7 Lendület grant of the Hungarian Academy of Sciences.

Funding for the Sloan Digital Sky Survey IV has been provided by the Alfred P. Sloan Foundation, the U.S. Department of Energy Office of Science, and the Participating Institutions. SDSS-IV acknowledges support and resources from the Center for HighPerformance Computing at the University of Utah. The SDSS web site is www.sdss.org.

\section{DATA AVAILABILITY}

The data underlying this article are available in https://github.com/Hydralisk24/Science

\section{REFERENCES}

Albareti F. D. et al., 2017, ApJS, 233, 25

Arnett W. D., 1980, ApJ, 237, 541A

Arnett W. D., 1982, ApJ, 253, 785A

Arnett W. D., Fu A., 1989, ApJ, 340, 396

Baklanov P. V., Blinnikov S. I., Pavlyuk N. N., 2005, AstL, $31,429 \mathrm{~B}$

Bayless A. J. et al., 2013, ApJ, 764L, 13B

Benetti S. et al., 2001, MNRAS, 322, 361B

Blinnikov S. \& Popov D. V., 1993, A\&A, 274, 775

Blondin S., Tonry J. L., 2007, ApJ, 666, 1024B

Bose S. et al., 2013, MNRAS, 433, 1871B

Bottinelli L., Gouguenheim L., Paturel G., de Vaucouleurs G., 1984, A\&AS, 56, 381B

Bottinelli L., Gouguenheim L., Paturel G., Teerikorpi P., 1986, A\&A, 156, 157B

Brown P. J. et al., 2007, ApJ, 659, 1488B

Burrows A., 2013, RvMP, 85, 245B

Burrows A., Radice D., Vartanyan D., 2019, MNRAS, 485, 3153B

Chugai N. N., \& Utrobin V. P., 2000, A\&A, 354, 557

Chatzopoulos E.; Wheeler J. C., Vinko J., 2012, ApJ, 746, $121 \mathrm{C}$

Clocchiatti A., Wheeler J. C., 1997, ApJ, 491, 375C

Dall'Ora M. et al., 2014, ApJ, 787, 139D

Dessart L., Hillier D. J., 2005, A\&A, 439, 671D

Dessart L. et al., 2008, ApJ, 675, 644D

Eastman R. G., Schmidt B. P., Kirshner R., 1996, ApJ 466, 911

Eldridge J. J., Mattila S., Smartt S. J., 2007, MNRAS, 376, L.52

Elmhamdi A. et al., 2003, MNRAS, 338, 939E

Fisher A., Branch D., Nugent P., Baron E., 1997, ApJ, 481, L89

Fixsen D. J., Cheng E. S., Gales J. M., Mather J. C., Shafer R. A., Wright E. L., 1996, ApJ, 473, 576F

Fraser M. et al., 2011, MNRAS, 417., 1417F

Fraser M. et al., 2012, ApJ, 759L, 13F

Gal-Yam A. et al., 2011, ApJ, 736, 159

Gilks W. R., Richardson S., Spiegelhalter D. J., Markov Chain Monte Carlo in Practice (Chapman \& Hall: London, 1996)

Gutierrez C. P. et al., 2017, ApJ, 850, 89G

Hamuy M., 2003, ApJ, 582, 905H

Hamuy M. et al. 2001, ApJ, 558, 615 (H01)

Hamuy M., Pinto P. A., 2002, ApJ, 566L, 63H

Hastings W. K., 1970, Biometrika, 57, 97

Hatano K., Branch D., Fisher A., Millard J., Baron E., 1999, ApJS, 121, 233H

Jerkstrand A., Ertl T., Janka H.-T., Müller E., 2017, MNRAS, Vol. 88, 278 
Jordi K., Grebel E. K., Ammon K., 2006, A\&A, 460, 339J

Kirshner R. P., Kwan J., 1974, ApJ, 193, 27

Kitaura F. S., Janka H.-Th., Hillebrandt W., 2006, A\&A, 450,345

Leonard D. C. et al., 2002, PASP, 114, 35L

Leonard D. C., Kanbur S. M., Ngeow C. C., Tanvir N. R., 2003, ApJ, 594, 247.

Li W. et al., 2006, ApJ, 641, 1060

Lisakov S. M., Dessart L., Hillier D. J., Waldman R., Livne E., 2018, MNRAS, 473, 3863L

Litvinova Y., Nadyozhin D. K., 1985, SvA Lett., 11, 45

Lyman J. D., Bersier D., James P. A., 2014, MNRAS, 437, $3848 \mathrm{~L}$

Maguire K. et al., 2010, MNRAS, 404, 981M

Mattila S., Smartt S. J., Eldridge J. J., Maund J. R., Crockett R. M., Danziger I. J., 2008, ApJ, 688, L91

Maund J. R., Smartt S. J., Danziger I. J., 2005, MNRAS, $364, \mathrm{~L} 33$

Metropolis N., Rosenbluth A. W., Rosenbluth M. N., Teller A. H., Teller E., 1953, J.Chem.Phys., 21, 1087

Misra K., Pooley D., Chandra P., Bhattacharya D., Ray A. K., Sagar R., Lewin W. H. G., 2007, MNRAS, 381, 280M

Morozova V. S. et al., 2015, ApJ, 814, 63

Mould J. R. et al., 2000, ApJ, 529, 786M

Nadyozhin D. K., 2003, MNRAS, 346, 97

Nagy A. P., Ordasi A., Vinko J., Wheeler J. C., 2014, A\&A, 571, A77

Nagy A. P., Vinko J., 2016, A\&A, 589, A53

Nagy A. P., 2018, ApJ, 862, 143N

Nakaoka T. et al., 2018, ApJ, 859, 78N

Pastorello A. et al., 2004, MNRAS, 347, 74

Pastorello A. et al., 2006, MNRAS, 370, 1752P

Pastorello A. et al., 2009, MNRAS, 394, 2266P

Planck Collaboration, 2018, A\&A, in press, arXiv:1807.06209

Popov D. V., 1993, ApJ, 414, 712

Poznanski D., Prochaska J. X., Bloom J. S., 2012, MNRAS, $426,1465 \mathrm{P}$

Pumo M. L., Zampieri L., Spiro S., Pastorello A., Benetti S., Cappellaro E., Manico G., Turatto M., 2017, MNRAS, 464, 3013P

Riess A. G., 2020, Nat. Rev. Phys. 2, 10

Roy R. et al., 2011, ApJ, 736, 76

Sahu D. K., Anupama G. C., Srividya S., Muneer S., 2006, MNRAS, 372, 1315S

Schlafly E.F., Finkbeiner P. D., 2011, ApJ 737, 103

Seitenzahl I. R., Timmes F. X., Magkotsios G., 2014, ApJ, 792, 10S

Smartt S. J., Eldridge J. J., Crockett R. M., Maund J. R., 2009, MNRAS, 395, 1409

Spiro S. et al., 2014, MNRAS, 439, 2873S

Takats K., Vinko J., 2006, MNRAS, 372, 1735T

Takats K. et al., 2014, MNRAS, 438, 368T

Thomas R. C., Nugent P. E., Meza J. C., 2011, PASP, 123, 237

Tomasella L. et al., 2015, ATel, 7787, $1 \mathrm{~T}$

Tully R. Brent, Fisher J. R., 1988, Cambridge University Press, cng, book T

Turatto M. et al., 1998, ApJ, 498, L129

Utrobin V. P., 2007, A\&A, 461, 233U

Utrobin V. P., Chugai N. N., 2008, A\&A, 491, 507

Utrobin V. P., Chugai N. N., 2009, A\&A, 506, 829U

Van Dyk S. D. et al., 2012, ApJ, 756, 131V

Wanajo S., Nomoto K., Janka H.-Th., Kitaura F. S., Mueller B., 2009 ApJ 695, 208

Woosley S. E., Hartmann D., Pinto P. A., 1989, ApJ, 346, $395 \mathrm{~W}$ 


\section{APPENDIX}

In the Appendix we present a more detailed description about our fitting code, then show the results of fitting the LCs of several other well-known Type II-P SNe, and compare them with the published parameter values for these $\mathrm{SNe}$ that were derived using various methods.

First, the numerical routines within the modeling part have been improved in order to make the running time as short as possible. This was an essential step, as the MCMC method needs to run the code hundred thousands or millions of times to give sensible results. The algorithm which determines the ionization zone was also optimized.

The light curve is computed from three main components: $L, L_{\text {ion }}$, and $L_{\text {pos }}$ (see Nagy et al. 2014; Nagy \& Vinko 2016 for more details). $L$ is fully numerical at first, and computed with a fourth order RungeKutta method (for the differential equation see Eq. (12) of Nagy et al. 2014):

$\frac{d \phi(t)}{d t} \tau_{N i}=\frac{R(t)}{R_{0} \cdot x_{\mathrm{i}}^{3}} \cdot\left(p_{1} \zeta(t)-p_{2} x_{\mathrm{i}} \cdot \phi(t)-2 \tau_{N i} \cdot \phi(t) \cdot \frac{R_{0}}{R(t)} \frac{d x_{\mathrm{i}}}{d t}\right)$

$\zeta(t)$ is the energy coming from the ${ }^{56} \mathrm{Co}$ and ${ }^{56} \mathrm{Ni}$ decay, and $\tau_{N i}$ is the decay time of the nickel. $\tau_{d}$ is the diffusion timescale (Arnett 1980), $p_{1}$ and $p_{2}$ are constants (see Nagy et al. 2014).

$L+L_{\mathrm{ion}}=x_{\mathrm{i}} \cdot \frac{\phi(t) E_{\mathrm{th}}(0)}{\tau_{d}} \cdot\left(1-e^{-A_{g} / t^{2}}\right)+4 \pi r_{\mathrm{i}}^{2} Q \cdot \rho\left(x_{\mathrm{i}}, t\right) \cdot \frac{d r_{\mathrm{i}}}{d t}$

where $Q$ is the recombination energy per unit mass, $\rho$ is the density. $L_{\text {ion }}$ (the luminosity from ionization/recombination, $L+L_{\text {ion }}$ is equal with Eq. (15) of Nagy et al. (2014). Eq. (5) is semi-analytic: the $d r_{\mathrm{i}}$ component (layer width of the ionization zone) is calculated numerically, within the fourth order RungeKutta method. The ionization zone $\left(x_{\mathrm{i}}\right)$ is computed in every Runge-Kutta step, so $d r_{\mathrm{i}}$ becomes far more accurate in this way. $L_{\text {pos }}$ (luminosity from positrons, Woosley et al. 1989; Seitenzahl et al. 2014) is fully analytic.

The analytic nebular phase can be fitted separately. This be can be done because there are only two parameters that describe the nebular phase: the nickel mass $M_{\mathrm{Ni}}$ and the effective gamma-ray trapping $T_{0}$ (Clocchiatti \& Wheeler 1997). We use the parameter $A_{g}=T_{0}^{2}$ instead (Chatzopoulos et al. 2012). Because this part is analytical, it is fast. From this, the set of best parameter pairs $\left(A_{g}, M_{\mathrm{Ni}}\right)$ is determined in the form of a function $M_{\mathrm{Ni}}\left(A_{g}\right)$ which describes what nickel mass fits the nebular phase for various values of $A_{g}$.

$A_{g}$ has the following form (Clocchiatti \& Wheeler 1997):

$$
A_{g}=T_{0}^{2}=\frac{\kappa_{\gamma} \cdot M_{\mathrm{ej}}}{4 \cdot \pi \cdot f \cdot v^{2}},
$$

where $\kappa_{\gamma}$ is the gamma-ray opacity, $f$ is a geometric factor, $g_{1}$ is a geometric integral (see Nagy \& Vinko 2016 for more information), and

$$
v^{2}=\frac{2 \cdot E_{\mathrm{kin}} \cdot f}{g_{1} \cdot M_{\mathrm{ej}}}
$$

So $E_{\text {kin }}$, and $M_{\mathrm{ej}}$ ( $\kappa_{\gamma}$ is not sampled) determine $A_{g}$, then $M_{\mathrm{Ni}}$ can be inferred from $M_{\mathrm{Ni}}\left(A_{g}\right)$ function to ensure that the nebular phase is fitted as closely as possible.

The physical equations and methods remained identical with those used in LC2.2. Also, the upgraded version has less numerical instabilities. Because of this, the outputs of the two models - the original LC2.2 and the new LC3.2 - are essentially the same.

The parameters in our case are the initial radius $\left(R_{0}\right)$, the ejected mass $\left(M_{\mathrm{ej}}\right)$, and the energies (total explosion energy: $E_{0}=E_{\text {kin }}+E_{\mathrm{th}}$, kinetic: $E_{\text {kin }}$, thermal: $\left.E_{\text {th }}\right)$.

All of them are set as scale parameters. We adopted uniform a priori pdf-s in their logarithms, which corresponds to a regularized Jeffreys prior. In fact, we directly sampled their logarithms in the algorithm rather than the parameters themselves.

The reported parameter values are to the mode of the joint posterior, corresponding to the best fitting solution. The upper and lower uncertainty limits are derived from the $2 \sigma$ (0.95 level) confidence intervals of the marginalized pdf around the best solution.

In the MCMC the likelihood correlates with the sample number, so this simplifies the calculation, as only the sample number needs to be plotted, and the sample mean, standard deviation, measure of correlations and confidence intervals can be computed by simple summation over the chain elements.

As a result, we find good match between our results and those of others. Details are given below.

The posterior distribution and best fits for the reference SNe are shown in (Fig. 12) and in (Fig. 13). The values in Table $6-9$. Because of the correlations between various parameters, and the usage of various method gives a slightly different values in the literature. The radius has a very strong correlation with the thermal energy giving very high uncertainty. The mass values are more or less consistent. The advantage of an MCMC sampling over simple optimization methods is that it also allows the consistent assesment of the parameter uncertainties. Considering this, we are in good agreement with previous results by Nagy \& Vinko (2016) and others. The energy median is somewhat larger, but the literature values are within our lower error limits. The other parameters are in very good agreement with the literature.

There are estimation formulae (Litvinova \& Nadyozhin 1985; Nadyozhin 2003) to derive ejected mass, radius and energy from the light-curve shape without modeling it. This is often used in literature, but may have large uncertainties. The values inferred this method appear in the Tables in columns labeled as 'formulae'. Note: $v_{\exp }$ is not 


\begin{tabular}{lcccccc}
\hline Parameter & LC3.2 BB $2 \sigma$ & LC3.2 RJ $2 \sigma$ & Bose & Utrobin & Elmhandi & Baklanov \\
\hline Method & $\begin{array}{c}\text { semi- } \\
\text { analytic }\end{array}$ & $\begin{array}{c}\text { semi- } \\
\text { analytic }\end{array}$ & $\begin{array}{c}\text { semi- } \\
\text { analytic }\end{array}$ & hydro & formulae & formulae \\
\hline$R_{0}\left[10^{11} \mathrm{~cm}\right]$ & $659_{+37}^{-639}$ & $52_{+645}^{-32}$ & $280 \pm 38$ & $350 \pm 140$ & $95 \pm 11$ & $508 \pm 193$ \\
$M_{\mathrm{ej}}\left[\boldsymbol{M}_{\odot}\right]$ & $19.10_{+3.57}^{-6.18}$ & $13.16_{+4.94}^{-2.04}$ & $11 \pm 3$ & - & $10-11$ & $16.5 \pm 1.5$ \\
$M_{0}\left[\boldsymbol{M}_{\odot}\right]$ & - & - & - & $19 \pm 1.2$ & - & - \\
$E_{\mathrm{kin}}\left[10^{51} \mathrm{erg}\right]$ & $4.21_{+4.10}^{-1.55}$ & $2.52_{+2.79}^{-0.78}$ & - & - & - & - \\
$E_{\mathrm{th}}\left[10^{51} \mathrm{erg}\right]$ & $0.32_{+9.98}^{-0.05}$ & $3.47_{+4.85}^{-3.21}$ & - & - & - & - \\
$E_{0}\left[10^{51} \mathrm{erg}\right]$ & - & - & $0.5-0.9$ & 1.3 & $0.5-1$ & $0.7-1$ \\
$E_{\mathrm{th}} R_{0}$ & $210_{-29}^{+63}$ & $179_{-36}^{+24}$ & - & - & - & - \\
$\cdot\left[10^{62} \mathrm{erg} \mathrm{cm}\right]$ & $6078_{-455}^{+1865}$ & $5658_{-646}^{+1025}$ & - & - & - & - \\
$v_{\mathrm{exp}}\left[\mathrm{km} \mathrm{s} \mathrm{km}^{-1}\right]$ & 0.06 & 0.04 & - & 0.036 & 0.02 & - \\
$M_{\mathrm{Ni}}\left[\boldsymbol{M}_{\odot}\right]$ & $0.2: 0.3$ & $0.2: 0.3$ & - & - & - & - \\
$\kappa\left[\mathrm{cm}^{2} \mathrm{~g}^{-1}\right]$ & & & & & & \\
\hline
\end{tabular}

Table 6. SN 1999em fit and literature values. Mass before explosion: $M_{0}$, Full energy: $E_{0}$. Bose: Bose et al. 2013; Utrobin: Utrobin 2007; Elmhandi: Elmhamdi et al. 2003; Baklanov: Baklanov et al. 2005 *: Not identical velocity with our velocities. $s=0$.

identical with photometric velocities, see Nagy \& Vinko ( 2016).

There are existing pre-explosion Hubble Space Telescope (HST) images which give independent observation values for the $\mathrm{SNe}$ parameters: 2005cs: $M_{0}=6-13 \quad M_{\odot}$ (Maund et al. 2005; Li et al. 2006; Eldridge et al. 2007). 2004et: Mass before explosion: $M_{0}=8-14 M_{\odot}$ (Smartt et al. 2009). 2012aw: $M_{0}=17-18 M_{\odot}($ Van Dyk et al. 2012), and $M_{\mathrm{ZAMS}}=14-26 M_{\odot}$ and $R_{0}>350 \cdot 10^{11} \mathrm{~cm}$ (Fraser et al. 2012).

Our code presented in this paper can be downloaded from:

https://github.com/Hydralisk24/Science/tree/master/SN-LC-MCMC

Contact: jagerz24@gmail.com 


\begin{tabular}{lccccccc}
\hline Parameter & LC3.2 2 $\sigma$ & LC3.2 2 $\sigma$ & $\begin{array}{c}\text { Nagy, } \\
\text { Vinko }\end{array}$ & Sahu & Bose & Utrobin & Misra \\
\hline Method & $\begin{array}{c}\text { semi- } \\
\text { analytic }\end{array}$ & $\begin{array}{c}\text { semi- } \\
\text { analytic }\end{array}$ & $\begin{array}{c}\text { semi- } \\
\text { analytic }\end{array}$ & formulae & formulae & hydro & formulae \\
\hline$R_{0}\left[10^{11} \mathrm{~cm}\right]$ & $306_{-284}^{+424}$ & $26_{-2}^{+703}$ & 420 & - & $414 \pm 63$ & $1050 \pm 98$ & - \\
$M_{\mathrm{ej}}\left[M_{\odot}\right]$ & $12.99_{-4.11}^{+2.59}$ & $17.76_{-7.43}^{+1.76}$ & 11.0 & $15 \pm 5$ & $9 \pm 2$ & - & $12 \pm 4$ \\
$M_{0}\left[M_{\odot}\right]$ & - & - & - & - & - & $24.5 \pm 1$ & - \\
$E_{\mathrm{kin}}\left[10^{51} \mathrm{erg}\right]$ & $3.14_{-1.40}^{+1.56}$ & $3.85_{-1.85}^{+1.20}$ & 1.35 & - & - & - & - \\
$E_{\mathrm{th}}\left[10^{51} \mathrm{erg}\right]$ & $1.02_{-0.63}^{+10.1}$ & $11.7_{-11.3}^{+0.19}$ & 0.60 & - & - & - & - \\
$E_{0}\left[10^{51} \mathrm{erg}\right]$ & - & - & 1.95 & $1.24 \pm 0.34$ & $0.6 \pm 0.2$ & $2.3 \pm 0.3$ & $1.96 \pm 0.25$ \\
$E_{\mathrm{th}} R_{0}$ & $312_{-84}^{+32}$ & $307_{-64}^{+38}$ & - & - & - & - & - \\
.$\left[10^{62} \mathrm{erg} \mathrm{cm}\right]$ & & & & - & - & $*$ & - \\
$v_{\mathrm{exp}}\left[\mathrm{km} \mathrm{s}^{-1}\right]$ & $6361_{-1052}^{+322}$ & $6029_{-720}^{+654}$ & 4250 & - & - & 0.068 & 0.06 \\
$M_{\mathrm{Ni}}\left[M_{\odot}\right]$ & 0.06 & 0.06 & 0.06 & 0.06 & - & - & - \\
$\kappa\left[\mathrm{cm}^{2} \mathrm{~g}^{-1}\right]$ & 0.3 & $0.2: 0.3$ & 0.3 & - & - & - \\
\hline
\end{tabular}

Table 7. SN 2004et LC fit and literature values. Mass before explosion: $\boldsymbol{M}_{0}$, Full energy: $\boldsymbol{E}_{0}$. Nagy, Vinko: Nagy \& Vinko 2016 ; Sahu: Sahu et al. 2006; Bose: Bose et al. 2013; Utrobin: Utrobin \& Chugai 2009; Misra: Misra et al. 2007. *: Not identical velocity with our velocities. $s=0$.

\begin{tabular}{lccccccc}
\hline Parameter & LC3.2 $2 \sigma$ & LC3.2 $2 \sigma$ & Nagy & Utrobin & Pastorello & Takats & Spiro \\
\hline Method & $\begin{array}{c}\text { semi- } \\
\text { analytic }\end{array}$ & $\begin{array}{c}\text { semi- } \\
\text { analytic }\end{array}$ & $\begin{array}{c}\text { semi- } \\
\text { analytic }\end{array}$ & hydro & $\begin{array}{c}\text { semi- } \\
\text { analytic }\end{array}$ & formulae & hydro \\
\hline$R_{0}\left[10^{11} \mathrm{~cm}\right]$ & $218_{-198}^{+218}$ & $33_{-13}^{+158}$ & 120 & $420 \pm 98$ & 70 & $180 \pm 126$ & 250 \\
$M_{\mathrm{ej}}\left[\boldsymbol{M}_{\odot}\right]$ & $8.84_{-1.19}^{+1.10}$ & $8.80_{-0.86}^{+1.52}$ & 8.00 & - & $11 \pm 3$ & $9.7 \pm 5.4$ & 9.5 \\
$M_{0}\left[\boldsymbol{M}_{\odot}\right]$ & - & - & - & $17 \pm 1$ & - & - & - \\
$E_{\mathrm{kin}}\left[10^{51} \mathrm{erg}\right]$ & $0.62_{-0.14}^{+0.17}$ & $0.62_{-0.14}^{+0.11}$ & 0.32 & - & - & - & - \\
$E_{\mathrm{th}}\left[10^{51} \mathrm{erg}\right]$ & $0.11_{-0.01}^{+0.94}$ & $0.71_{-0.60}^{+0.27}$ & 0.16 & - & - & - & - \\
$E_{0}\left[10^{51} \mathrm{erg}\right]$ & - & - & 0.48 & 0.41 & 0.3 & $0.23 \pm 0.14$ & 0.16 \\
$E_{\mathrm{th}} R_{0}\left[10^{62} \mathrm{erg} \mathrm{cm}\right]$ & $23.8_{-20}^{+0.7}$ & $23.5_{-5.2}^{+0.2}$ & - & - & - & - & - \\
$v_{\mathrm{exp}}\left[\mathrm{km} \mathrm{s} \mathrm{s}^{-1}\right]$ & $3441_{-279}^{+107}$ & $3444_{-459}^{+94}$ & 2580 & $*$ & $*$ & - & $*$ \\
$\boldsymbol{M}_{\mathrm{Ni}}\left[10^{-3} \boldsymbol{M}_{\odot}\right]$ & 2.8 & 2.8 & 2 & 8.2 & 3 & 3 & $6 \pm 3$ \\
$\kappa\left[\mathrm{cm}^{2} \mathrm{~g}^{-1}\right]$ & 0.3 & $0.2: 0.3$ & 0.3 & - & - & - & - \\
\hline
\end{tabular}

Table 8. SN 2005cs fit and literature values. Mass before explosion: $M_{0}$, Full energy: $E_{0}$. Nagy: Nagy \& Vinko 2016; Utrobin: Utrobin \& Chugai 2008; Pastorello: Pastorello et al. 2009; Takats: Takats \& Vinko 2006; Spiro: Spiro et al. 2014. *: Not identical velocity with our velocities. $s=0$.

\begin{tabular}{lccccc}
\hline Parameter & LC3.2 $2 \sigma$ & LC3.2 $2 \sigma$ & Nagy, Vinko & Bose & Dall'ora \\
\hline Method & semi-analytic & semi-analytic & semi-analytic & formulae & radiation hydro \\
\hline$R_{0}\left[10^{11} \mathrm{~cm}\right]$ & $579_{-559}^{+117}$ & $286_{-265}^{+476}$ & 295 & $337 \pm 67$ & 300 \\
$M_{\mathrm{ej}}\left[M_{\odot}\right]$ & $14.92_{-3.37}^{+2.51}$ & $14.54_{-1.62}^{+6.49}$ & 20.0 & $14 \pm 5$ & 20 \\
$E_{\mathrm{kin}}\left[10^{51} \mathrm{erg}\right]$ & $3.07_{-1}^{+1.00}$ & $2.98_{-0.98}^{+1.73}$ & 1.60 & - & - \\
$E_{\mathrm{th}}\left[10^{51} \mathrm{erg}\right]$ & $0.47_{-0.11}^{+11.4}$ & $0.92_{-0.58}^{+8.67}$ & 0.60 & - & - \\
$E_{0}\left[10^{51} \mathrm{erg}\right]$ & - & - & 2.20 & $1-2$ & 1.5 \\
$E_{\mathrm{th}} R_{0}\left[10^{62} \mathrm{erg} \mathrm{cm}\right]$ & $271_{-55}^{+18}$ & $264_{-48}^{+25}$ & - & - & - \\
$\nu_{\exp }\left[\mathrm{km} \mathrm{s}^{-1}\right]$ & $5875_{-863}^{+434}$ & $5855_{-1123}^{+102}$ & 3650 & - & $*$ \\
$M_{\mathrm{Ni}}\left[M_{\odot}\right]$ & 0.06 & 0.06 & 0.06 & 0.06 & 0.06 \\
$\kappa\left[\mathrm{cm}^{2} \mathrm{~g}^{-1}\right]$ & 0.3 & $0.2: 0.3$ & 0.13 & - & - \\
\hline
\end{tabular}

Table 9. SN 2012aw fit and literature values. Mass before explosion: $M_{0}$, Full energy: $E_{0}$. Nagy, Vinko: Nagy \& Vinko 2016 ; Bose: Bose et al. 2013; Dall'ora: Dall'ora et al. 2014. *: Not identical velocity with our velocities. $s=0$. 

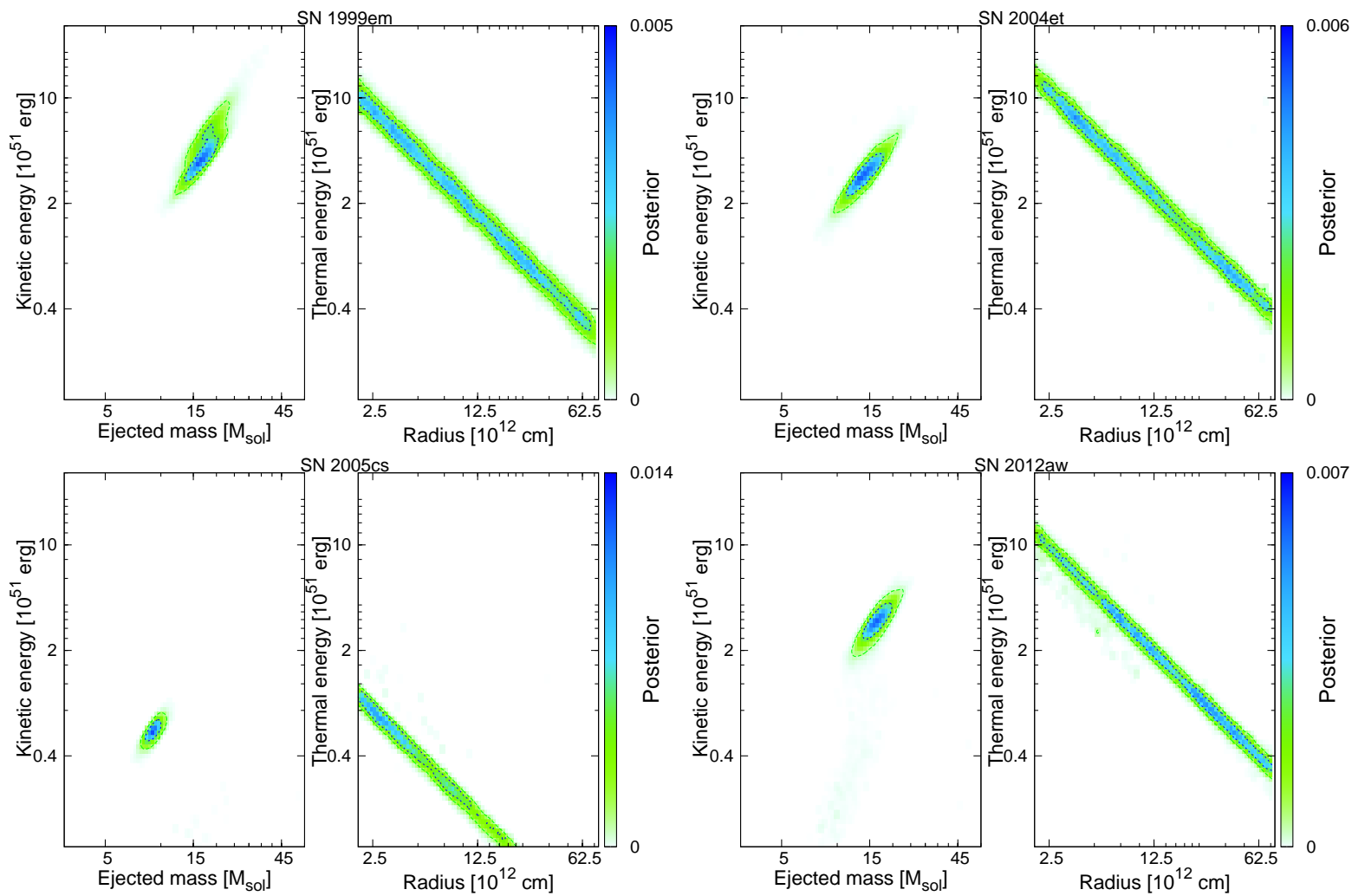

Figure 12. Other SNe probability distributions of the joint parameters, upper left: SN 1999em (BB fit; see text), upper right: SN 2004et, bottom left: SN 2005cs, bottom right: SN 2012aw. $s=0, \kappa=[0.2: 0.3] \mathrm{cm}^{2} \mathrm{~g}^{-1}$. The colors encode the likelihood as the goodness of the fits. The contours show the confidence interval of $67 \%$ and $95 \%$.
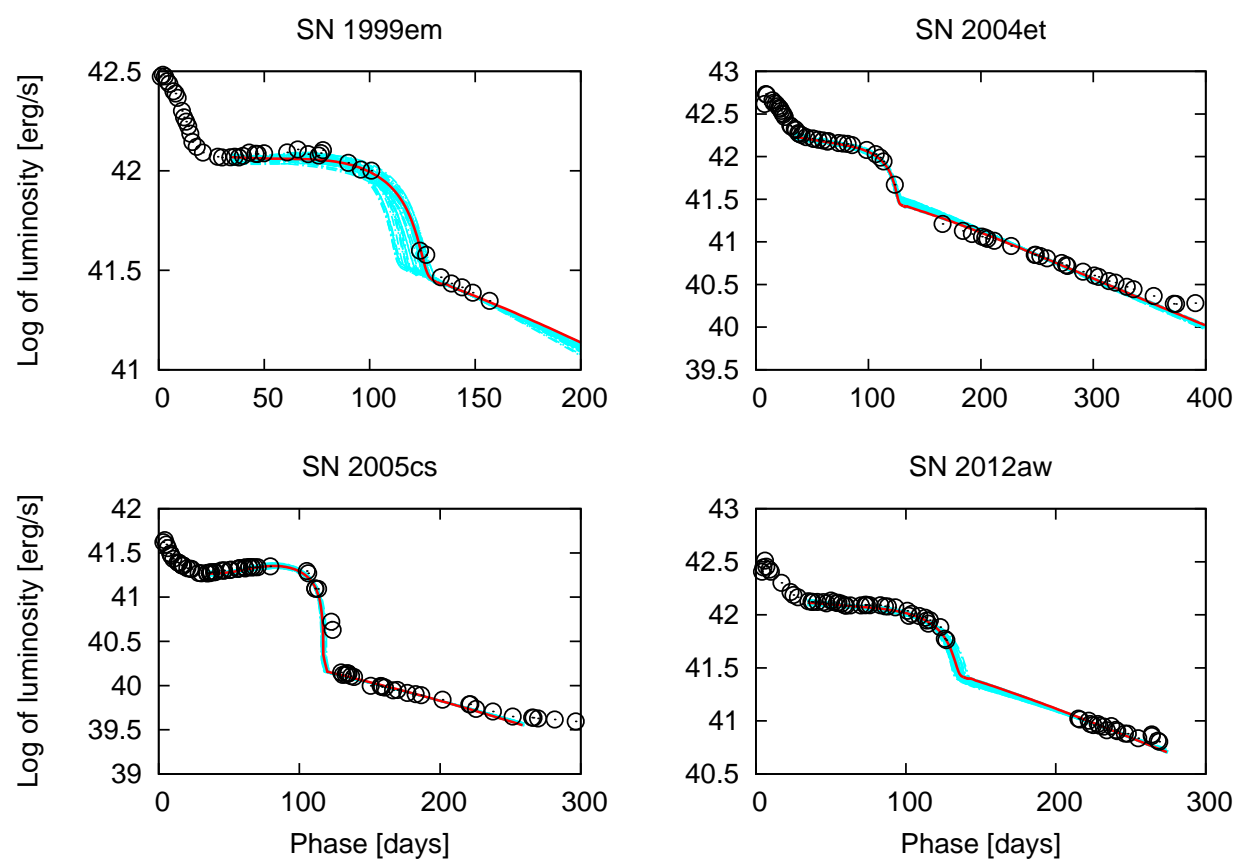

Figure 13. Model fits for the comparison SNe. The red line is the best fit. $s=0, \kappa=[0.2: 0.3] \mathrm{cm}^{2} \mathrm{~g}^{-1}$. (1999em: BB fit used; see text) 